\title{
Pattern operators for grid environments
}

\author{
Maria Cecília Gomes ${ }^{\mathrm{a}}$, Omer F. Rana ${ }^{\mathrm{b}}$ and José C. Cunha ${ }^{\mathrm{a}}$ \\ ${ }^{a}$ Departamento de Informática, Universidade Nova de Lisboa, Portugal \\ ${ }^{\mathrm{b}}$ Department of Computer Science, Cardiff University, UK
}

\begin{abstract}
A pattern based approach for developing applications in a Grid computing environment is presented, and is based on the ability to manage components and their interactions. The approach provides a formal way of combining recurrent themes in Grid applications, and provides a set of operators that may be used to manipulate the patterns. The operators may be applied to individual patterns or groups, and may be managed as an independent library. The patterns distinguish between service providers and users, and may be used to also analyse the properties of a collection of components, or to vary these properties subject to a set of predefined constraints. Patterns are expressed in the Unified Modelling Language (UML), and operators correspond to manipulation of components within each pattern.
\end{abstract}

\section{Introduction and related work}

Component based software development provides an effective way to develop applications from a range of different software libraries, and wrapped legacy codes. Components can vary in complexity and granularity ranging from complete applications to specialised subroutines. A number of projects (see a list in $[1,15]$ ) have explored component composition and workflow management for components in the context of Grid computing $[14,16]$. Generally, these environments involve a user interface which enables components to be selected from a repository, and combined using an editor. The interfaces to the components are generally pre-defined, and often expressed in XML - and these environments are generally called "Problem Solving Environments" (PSEs) [17]. Such environments generally consist of 3 tiers: i) a user portal to enable interaction with the components, (ii) a series of middle tier services - such as a data management service, one or more compute services, etc, and (iii) the physical resources on which the components are to be executed. Manipulating either individual components or groups of components is a useful extension - and little support is directly provided in existing environments to achieve this. It is also useful to determine and abstract common interactions between components, and to make these abstractions available to a user. One novel theme addressed in this paper is the ability to view component composition (to solve a particular problem) as being equivalent to manipulating a structural pattern using pre-defined operators. Subsequently, the resulting structure can be manipulated via behavioural operators that enable multiple data flows to co-exist within a system. A user (application developer) may find useful structural or behavioural patterns - in particular application contexts and record these within a patterns library. These can then be configured using the operator library.

The approach presented here is primarily aimed at computational scientists and developers, who have some understanding of the computational needs of their application domain. A scientist should be aware about the likely co-ordination and interaction types between components of the application (such as a database or numeric solver etc). The structural and behavioural patterns presented here will enable such scientists and developers to utilise common usage scenarios within a domain (either the use of particular components, such as database systems, or interactions between components, such as the use of streaming).

The features distinguishing Grid environments from other distributed computing approaches include: heterogeneity and dynamicity i.e. the infrastructure can change significantly over the lifetime of a single application, is composed of a range of different platforms, and may be managed by different administrators (see [21] for a useful survey). As there are likely to be a range of different users utilising a Grid infrastructure 
with differing abilities - less experienced users may find it difficult to identify useful architectural models for interconnecting components. The existence of a pre-defined set of patterns is therefore particularly useful in this context. Additionally, once components have been connected together, another major difficulty is the need to identify suitable coordination mechanisms between components. Providing a set of operators and common abstractions at the "behavioural" level is therefore important. This work aims to extend Grid application development environments with structuring mechanisms based on commonly recurring patterns. Using a library of design templates, a user is able to combine these with other specialised components that may be required in a particular application domain - both at design and execution times.

A number of approaches exist already for modelling interactions between components in the context of Grid environments, or for developing formal models of job submission and management in a Grid [29]. These, however, do not provide any support for enabling a user to subsequently utilise the outcome of these models. Marinescu [3,4] provides a common abstraction for modelling workflow to support Web and Grid Services. The approach is centered on developing graphical abstractions that can be used to model interaction patterns between components. The graphical patterns model aspects such as AND/OR/XOR based interactions - and the focus is to support a workflow enactment engine that may be used to co-ordinate component execution. Similarly, a key emphasis in the Fraunhofer Resource Grid [2] is on developing a Grid Resource and Job definition language, to enable job submission, resource selection, and allow a description of dependencies which exist between resources. In this work, the Grid Job Definition Language may be mapped to a series of parameterised Petri Net (PN) blocks. Each block represents some aspect of the language such as Task execution and synchronisation, Conditionals and Choice, and loops (such as the While ... do loop etc). Each PN block is encoded in XML based on the Petri Net Markup Language (PNML) [28]. Both of these approaches are focused on providing either a specialised representation scheme, or a workflow management approach for components and/or services. Our approach is more generic, and based on the provision of a standard pattern library in UML, and associated operators. Some of these operators may be used to support workflow, and PN models for patterns may also be constructed from their UML descriptions, as outlined in [6]. The PN models are useful to capture the seman- tics of the operators, and to undertake what-if investigations when combining operators. The availability of UML templates makes our approach more widely deployable, and may be used with a number of existing toolkits such as Rational Rose or TogetherJ (a survey can be found in [7]). The utilisation of languages such as Java (such as the CoG [12] interface to Globus) and emerging interest in Web Services [11] identifies the importance of using object-oriented design approaches. Various tools are currently available which can take UML diagrams and generate code fragments for these technologies. We therefore feel that a representation centered on UML is easier to translate into working designs.

Alternative related work has been undertaken by the parallel computing community, and is based on the use of algorithmic skeletons. The predominant motivation behind this has been the need to overcome the difficulty of constructing parallel programs - by capturing common algorithmic forms which may subsequently be used as components for building parallel programs [23, 24]. Such skeletons are expected to provide parameterisable abstractions that may be composed - generally using a functional programming language. A skeleton is expected to be transparent to an application user (and may come with a pre-packaged implementation). Skeletons are viewed formally as polymorphic, higher-order functions - which may be repeatedly applied to achieve various transformations (on data structures such as lists). Herrmann and Lengauer [25] outline the use of a programming language "Higher-order Divide and Conquer" (HDC) based on a subset of the functional programming language Haskell. They suggest that the use of a powerful type system in functional languages make them more suitable than other paradigms. Although useful for specifying programs in a concise syntax, we believe such approaches are limited in the context of Grid environments. This is primarily due to the absence of tools available in such languages for connecting to Grid middleware, such as Globus or UNICORE, although skeletons based approaches do provide a useful prototyping tool for analysis. Our use of "operators" (discussed in subsequent sections) borrows from the use of transformation techniques in skeleton based approaches, albeit our focus is on the use of object-oriented techniques. Furthermore, our design patterns and operators are aimed at supporting workflow-based PSEs.

A Pattern encodes a commonly recurring theme in service or component composition. It allows good practice to be identified, and shared across application 
domains. A pattern is generally defined in an application independent manner, and used to encode particular useful behaviours. Patterns are particularly useful for configuring and specifying systems that are composed of independent sub-domains. Patterns are aimed at capturing some generic attributes of a system - which may be further refined (eventually) to lead to an implementation. These are important requirements for Grid computing applications, which generally need to operate in dynamic environments. Providing patterns will ease the task of Grid application developers, who may deploy previously generated templates as an initial step, and then refine these based on our operators. The use of pattern operators is also particularly important to deal with dynamicity, because it provides a user the capability to modify a pattern at run time. Furthermore, pattern operators may be applied in an ordered combination and may be shared between users. The presented structural and behavioural operators may be implemented using a number of different scripting languages (such as Python or Perl) - and therefore the specified semantics are not restricted to our Java implementation.

The core of this work is the systematic identification of collections of operators that can manage a set of useful patterns in Grid environments, via a PSE. A PSE provides a collection of tools necessary to support problem description, and subsequently execution of the problem on computational resources. PSEs have ranged from those based on functional language based descriptions, to component-based composition tools. The component repository provides wrapped scientific codes or specialised components available within a given application domain - combined together using an editor, with access to a resource manager for execution. Patterns can be provided in a repository - and can include generic patterns (as discussed in this paper), or those created by a user.

Section 2 introduces an application example where patterns and operators may be useful for configuring and reconfiguring a PSE. This example will be used throughout the paper. Section 3 describes Pattern templates starting with Structural pattern templates and ending with Behavioural pattern templates. Section 4 describes the Structural and Behavioural Operators, and section 5 illustrates operator semantics. Section 6 describes implementation status. Finally, some conclusions are presented.

\section{An application example}

A PSE configuration example is provided to describe activities that are commonly required to manage an ap- plication. This example will be used throughout the paper for explaining the applicability of patterns and operators. Figure 1 shows an example of a PSE combining different types of services, which appear frequently in Grid applications. The Problem Solver component represents a service running some scientific experiment that continuously produces data. An instance of such a service may be a wave generator or a matrix solver. After receiving some initial input parameters, the service starts producing data that can be analysed or stored for "post-mortem" analysis. The Problem Solver service may be steerable, meaning that its input parameters can be changed while the service is executing. By adjusting the input parameters a user may, for example, generate particular behaviours using this service.

Steering is frequently supported by two types of services: a Monitoring Service and a Steering interface. The Monitoring Service is used to register relevant output data or events produced by the Problem Solver. The data/events are filtered by the Monitoring Service and are passed to a Steering Interface that shows them to the user in a pre-defined format. Consequently, a user may use the Steering Interface to undertake "what if" scenarios - generally by defining new values for the Problem Solver's input data. Furthermore, one may consider that several users have access to the Steering Interface, thus requiring some coordination over changing the parameters of the Problem Solver.

This type of application may also include another service, namely a Database System to store all the output produced by the Problem Solver. This enables a user to reconfigure the PSE using the stored data, without requiring the Problem Solver to be stopped. Alternatively, a user may re-examine output data for additional processing after the Problem Solver terminates its execution (based on pre-defined behaviour or as a result of a fault). These are illustrated in Figs 2 and 3: in Fig. 2 the Monitoring Service is stopped so that it can be replaced with a more complex tool like the Monitoring and Statistics service in Fig. 3; in the meantime, the Problem Solver continues its execution and its output is kept in the Database System. The alternative scenario is illustrated in Fig. 4: after the Problem Solver terminates its execution, its output can be processed, either from the beginning or from the point at which the Monitoring Service was being replaced (and that would otherwise be lost). In this case, the Database System acts as a temporary buffer.

The next section describes structural and behavioural pattern templates and identifies which patterns could be used to configure a PSE, as outlined in Fig. 1. Sec- 


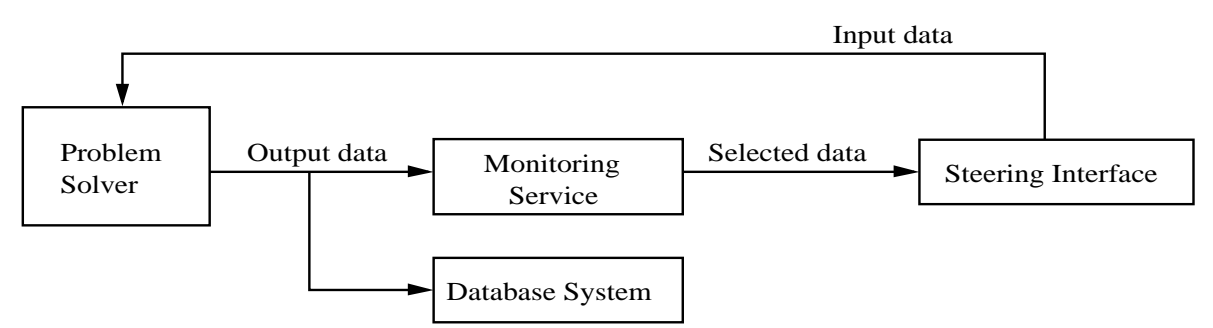

Fig. 1. A PSE supporting the active steering of a Problem Solver. The arrows represent the flow of data.

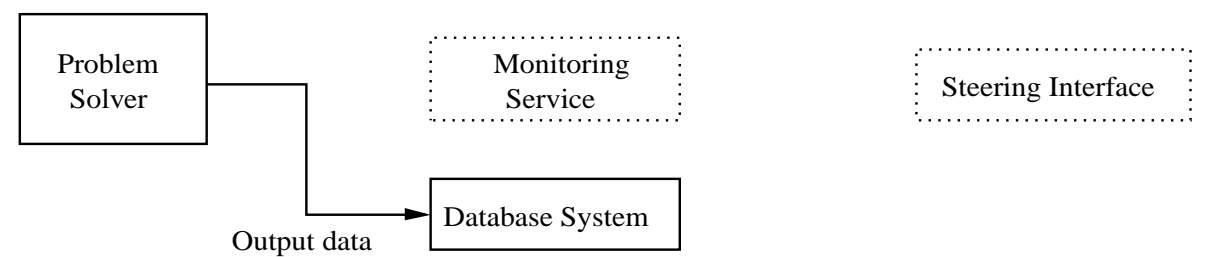

Fig. 2. The Monitoring service is stopped and consequently the Steering interface also stops. The output data is not lost because it is being saved in the Database system.

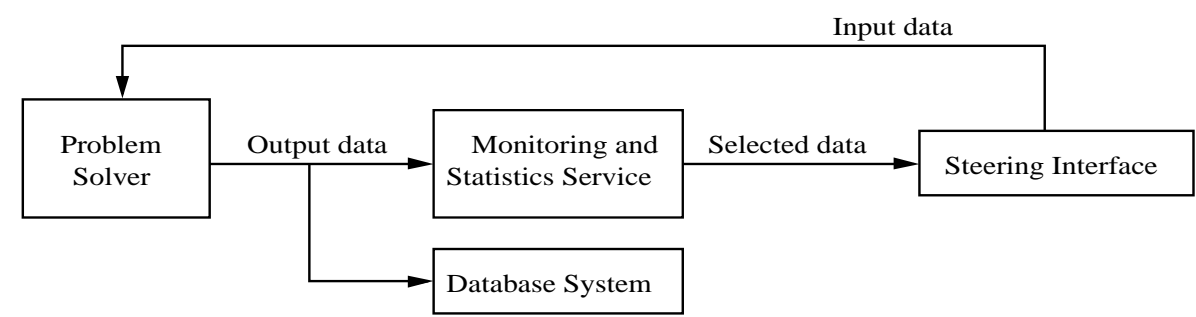

Fig. 3. The initial Monitoring service is replaced with a more complex one (Monitoring and Statistics service), which is activated to continue the filtering of the output data.

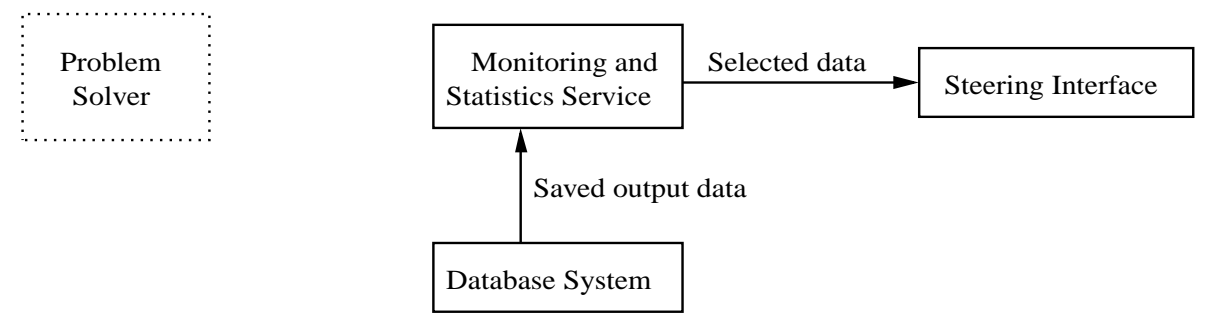

Fig. 4. After the Problem Solver terminates its execution, data can be re-analysed.

tion 4 subsequently describes the application of structural operators to build that PSE, and the application of behavioural operators to control the PSE's execution.

\section{Pattern templates}

Patterns capture commonly occurring structural and behavioural aspects of components. Structural patterns encode component connectivity, and identify common ways in which components may be combined within a given application domain (an example of this is the data flow pipeline used in rendering, which involves a data input, simulation/rendering, visualisation pipeline). Structural patterns may also contain a hierarchy, allowing the embedding of a pattern within another (these embedding are supported through specialised operators). Structural pattern templates therefore consist of component place holders, where each component is instantiated at run time. 
Behavioural patterns encode useful required functionality without necessarily identifying the particular components involved. Components within a behavioural pattern primarily identify interaction constraints, and not the exact functionality required from each component. Behavioural patterns can therefore capture temporal or flow dependencies between components. Flow dependencies model data and control flows, and encode execution ordering on components (flow dependencies may be used to express synchronisation constraints, for instance). Behavioural patterns may be defined based on interactions between components (such as Peer-2-Peer or Client/Server), to schemes used to update the behaviour of each component.

Our approach is applied at four different levels. The lowest level provides Structural Patterns - which enable static composition of components into a data/control flow. Structural Patterns enable the description of commonly recurring topological aspects of an application but do not constrain the flows between these topological entities in any way. These Structural Patterns may be manipulated via Structural Operators - which enable a constrained way to modify the Structural Patterns. The constraints are defined by the semantics of the operators - and relate to the result generated after the operator has been applied. Subsequently, flows on these Structural Patterns need to be specified - and this is achieved by identifying a Behavioural Pattern over the structure. Once again, the Behavioural Patterns may be configured statically or at run-time using Behavioural Operators. The division into these four stages of design is based on existing uses of application construction in PSEs. Based on our approach, a user must first commit to a structural pattern, and then to a behavioural one. Structural patterns therefore try to capture how many machines (for instance) or groups are necessary to execute a given application - and do not instantiate these to particular instances until the Behavioural operators are applied. The four stage approach therefore reflects the approach adopted by application schedulers - but tries to abstract this as a collection of patterns and operators - and brings it closer to the application construction process.

To use these patterns and operators, a user launches a PSE visual editor to connect components together. This PSE tool (as identified in [17]) is augmented with a Pattern Template (PT) and Operator library. The user may select a PT from the library, and may apply one or a combination of operators to modify the structure of the template. The structural operators provide a trans- formation between patterns, and are invariant to a given PT structure. The user can also modify the structure of a PT directly using the editor. The result may be stored by the user as a new template in a user-defined PT library. Once the structure has been defined, the user now instantiates components to the elements of a PT. This is then followed by defining interactions between components - based on the provided Behavioural Pattern Templates. Subsequently, these interactions may be modified using the Behavioural Operators.

The rest of this section gives examples of structural and behavioural patterns, as well as which could be used to configure the example defined in section 2 .

\subsection{Structural pattern templates: Topological}

Topological patterns represent structural shapes that frequently occur in Grid systems. For illustration purposes, we identify three basic shapes as possible candidates within this category: star, pipeline, and ring.

The Star pattern is an aggregation of three components: the Nucleus is the center of the star; a Satellite represents the elements communicating with the star; and the Simple Channel binds together a Satellite to the Nucleus. The Nucleus may be connected to several instances of SimpleChannel, but each SimpleChannel is only connected to a single Satellite. The Client/Server model, for example, is simply a specific behavioral pattern over the Star structural pattern.

The Pipeline pattern is a sequence of stages which communicate with each other. The pattern occurs frequently in Grid applications. For example, a scientific application produces data to a sequence of filters (like Data Analysis Tools), and the pipeline is terminated in a Visualisation Tool where the user can follow the application's execution. The pattern's structure was adapted from the Pipes and Filters pattern [9].

The structure can be generally represented by three components (see left-hand side of Fig. 5): a DataSource produces data to a Connector, and the DataSink consumes data from the Connector.

The Connector has a recursive structure, as illustrated in the right-hand side of Fig. 5. A Connector may be a SimpleConnector (similar to a Unix pipe or an event channel) or it may be a CompositeConnector. The latter is a connected association of a SimpleConnector and a Component. Recursively, the CompositeConnector is connected to another Connector (and terminates at the Simple Connector).

The Ring pattern represents, like the pipeline, a sequence of stages, with no "first" or "last" stage. The 

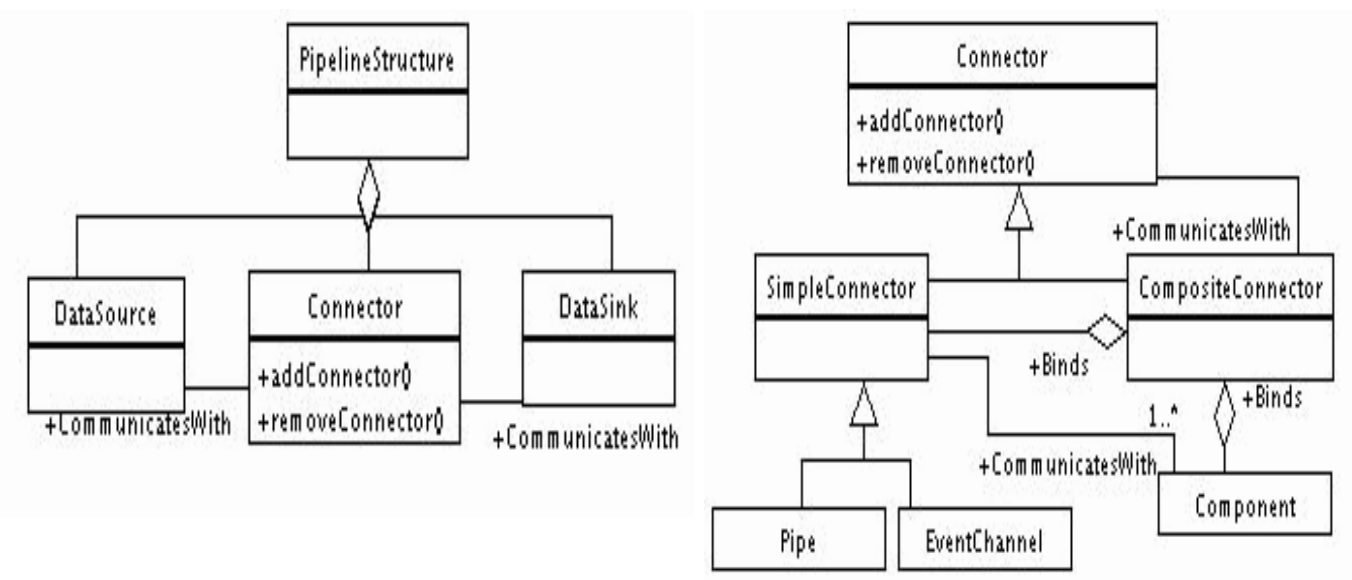

Fig. 5. The Pipeline and the Connector patterns.

structure of the Ring pattern is also based on the Connector structural definition.

The difference is that a Component is always connected to two SimpleConnectors (in the simplest case, the unique component will have two connections to a single SimpleConnector). In Grid environments, the ring topological structure can be found in a number of applications, both in the context of application execution (such as for modelling interactions within a local area network) to logical topologies such as supporting an authentication chain when approving participants with multiple Certificate server. Each server delegates an authentication request to the next domain, and the last server replies to the original client. This chain based mechanism can also be found in resolving the address/location of an executable using a directory lookup service (as found in the Globus MDS [10]).

\subsection{Structural pattern templates: Non-topological}

The Adapter, Facade, and Proxy design patterns (adapted from [8]) are examples of non-topological structural patterns, which are particularly useful in the context of Grid computing.

The Adapter pattern allows communication between two elements when they do not have the same interface. In the Grid environment, the Adapter pattern has applicability, for example, in the adaptation of services, or as wrappers for legacy codes (such as Fortran binaries). If the client is expecting a different interface from the one provided by the server, the adapter can act as a translator. This pattern is also particularly useful for providing a mapping between the interface of an existing code and a pre-defined component data model for Grids, such as CCA [26].
The Facade pattern (Fig. 6) is used when a system may be divided into several sub-systems, and the communication/entry-point into the system needs to be restricted. The Facade pattern is present in the structuring of the Grid in "domains". The access to each domain (sub-system) in the Grid may be via a Facade interface.

The Proxy pattern is frequent in distributed systems. The access to Grid services, for example, is usually through a proxy (or gatekeeper). The structure of the pattern consists of an abstract interface (the Subject) representing the service, the implementation of the service (RealSubject), and a surrogate (Proxy) which forwards the request to the implementor of the service.

\subsection{Structural patterns in use}

The PSE example from Fig. 1 can be configured based on some of the structural patterns described in the previous sections. As shown in Fig. 7, it is possible to identify a ring pattern connecting the Problem Solver, the Monitoring Service and the Steering Interface. There is also a pipeline pattern connecting the Problem Solver and the Database System. To represent such a PSE, the user would define a ring template with three elements, and a pipeline template with two elements. One way to combine the two patterns would be to embed the pipeline into one of the ring's elements forming an hierarchy.

Figure 8 identifies three more structural patterns, namely for configuring individual services. For example, the star pattern may represent the Database System considering that the system is composed of a set of distributed Database sub-systems. These sub-systems 


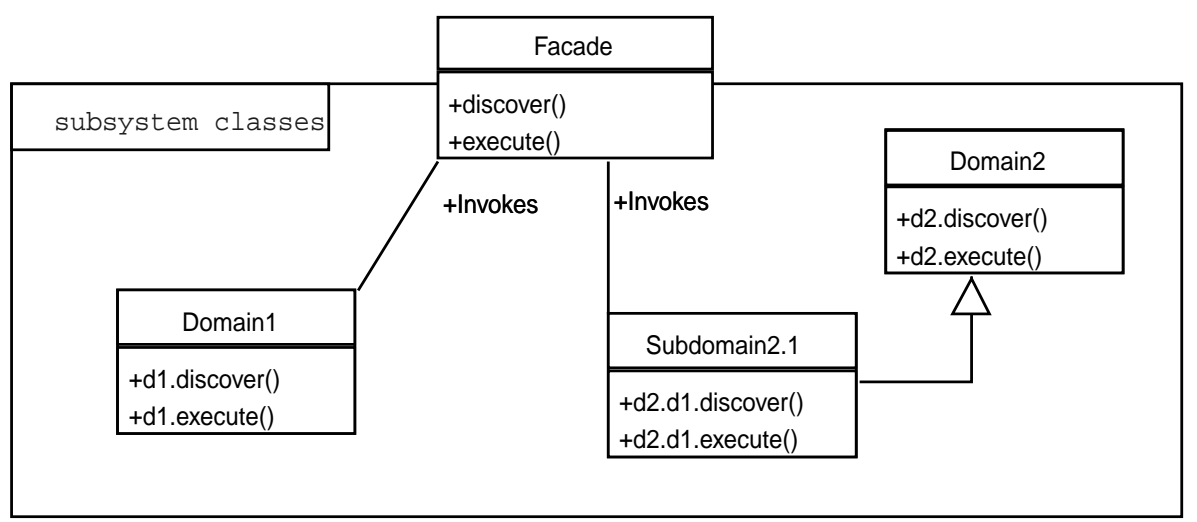

Fig. 6. The Facade design pattern. Example: the "Facade" provides a unified interface for accessing domains in the Grid environment, redirecting the calls to services like "discover" and "execute".

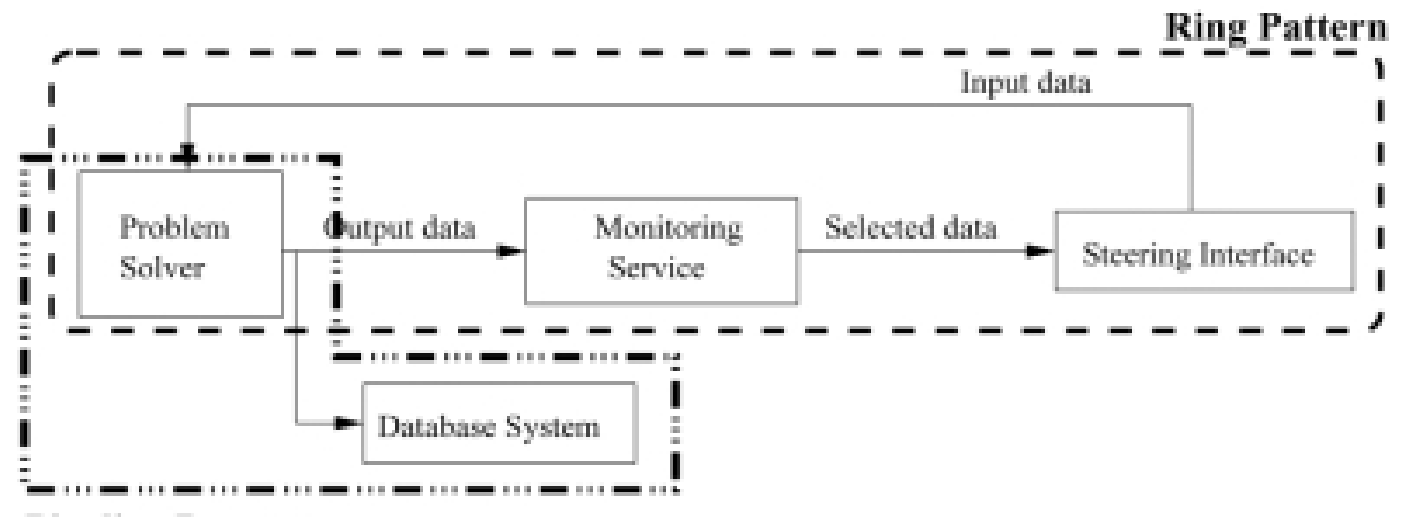

\section{Pipeline Pattern}

Fig. 7. Identification of the Ring and Pipeline patterns in the PSE example.

are the Satellites in the ring's structure, and they are controlled by a Master Database system acting as a coordinator. The figure shows a star template with three satellites. The second example in Fig. 8 shows the possibility of using an adapter pattern for the Monitoring service. This service may be supported by legacy code which needs to be adapted to interact with the other services. This adapter pattern would be embedded in the second element of the ring. Finally, one way to represent the sharing of the Steering Interface by multiple users is through the proxy pattern. Each user has a proxy to access the central service which controls concurrent accesses. The figure shows a proxy pattern template with two proxies for two users.

\subsection{Behavioural pattern templates}

Behavioural Pattern Templates (B-PT) capture recurring themes in component interactions within Grid applications. Generally, these applications involve distribution of code from a master, the replication of a code segment (such as within a loop), or parameter sweeps over one or more indices. The Parameter-Sweep template represents the repeated invocation of a component - over a range, and can be found in systems such as Nimrod [18]. The Master-Slave pattern can be mapped to many parallel programming libraries, and represents the division of a task into multiple (usually independent) sub-units - and shares some similarities with the Client-Server pattern - although the control flow in the latter is more complex.

Figure 9 illustrates the sequence diagram for the $\mathrm{Mo}$ bile Agent/Itinerary pattern. In this pattern a component is initialised at a given location (Home), and may move to another location based on a pre-defined itinerary - which may be defined using a structural pattern (for instance). If the itinerary is dynamic, then new locations may be created via the Increase, Extend 


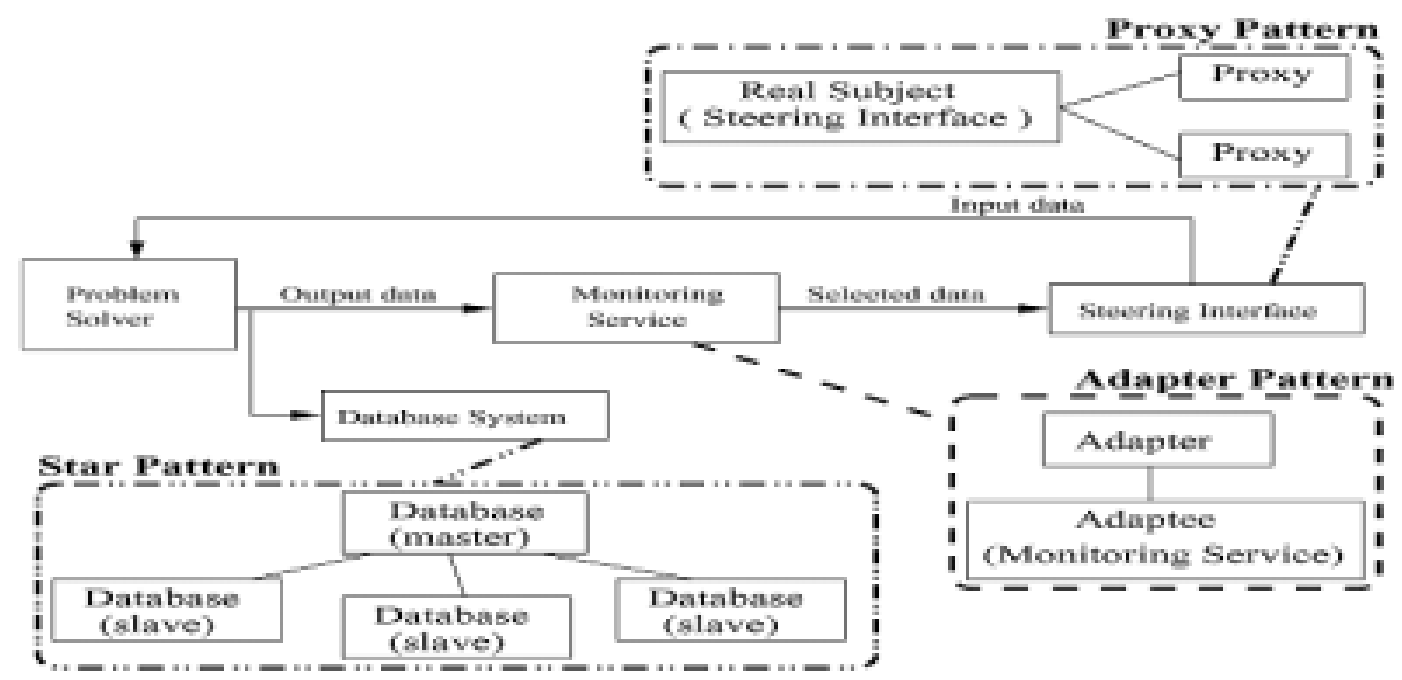

Fig. 8. Identification of the Facade, Adapter, and Proxy patterns in the PSE example.

or Embed operators. Each location that a mobile agent visits, is represented with a component place holder. Each of these can contain a Proxy pattern, to enable a chain of forwarders to be established.

\subsection{Behavioural patterns in use}

Taking as a basis the Structural Patterns illustrated in Figs 7 and 8 (section 3.3), this section enumerates some applicable behavioural patterns. See Table 1 in section 4.4 for a more complete list. Firstly, the Producer/Consumer pattern can be used to represent the control and data flows between the Monitoring service (producer of selected data) and the Steering Interface (consumer), in the ring pattern (Fig. 7). Secondly, the same pattern can represent the interaction between the Problem Solver and the Monitoring Service. However, if the Monitoring service only requires a sub-set of the data produced by the Problem Solver, then such interaction may be represented by the Observer pattern. Thirdly, the Streaming pattern may be used over the structural pipeline pattern that connects the Problem Solver and the Database system, representing the continuous flow of data from the Problem Solver that needs to be maintained in the Database System. Fourthly, the Master/Slave pattern can represent the behaviour of the Database System (Fig. 8): a master controls and distributes requests to the slaves. Fifthly, the Client/Server pattern can represent the interaction between the Steering Interface (server) and its proxies (clients) that redirect users' requests to access the Steering service. Finally, the Adapter structural pattern that gives access to the legacy code to support the Monitoring service can be combined with the Service Adapter Pattern [32]. This behavioural pattern "attaches additional properties or behaviours to an existing application to enable it to be invoked as a service".

Having identified the structural and behavioural patterns, in the following sections we describe the available operators and their application. In particular, we describe how structural operators are used to configure the application example described so far, and give a small example of the use of behavioural operators to control application execution.

\section{Operators}

Operators enable constrained manipulation of patterns by a developer, and provide a limited set of methods to achieve this. Operators provide transformations between patterns, albeit subject to a set of constraints. It is possible for a group of operators to be applied (with a particular ordering). Furthermore, operators may be combined, leading to "compound operators", although this is only allowed if operators from the same category are chosen - to ensure consistency of the result. Two kinds of operators exist within our approach: Structural Operators, and Behavioural Operators. These are further divided into the following categories, with each category implemented as a separate class library, and each operator being a method call within the library:

1. Structuring: These operators are used to modify the connectivity between components in a template. 


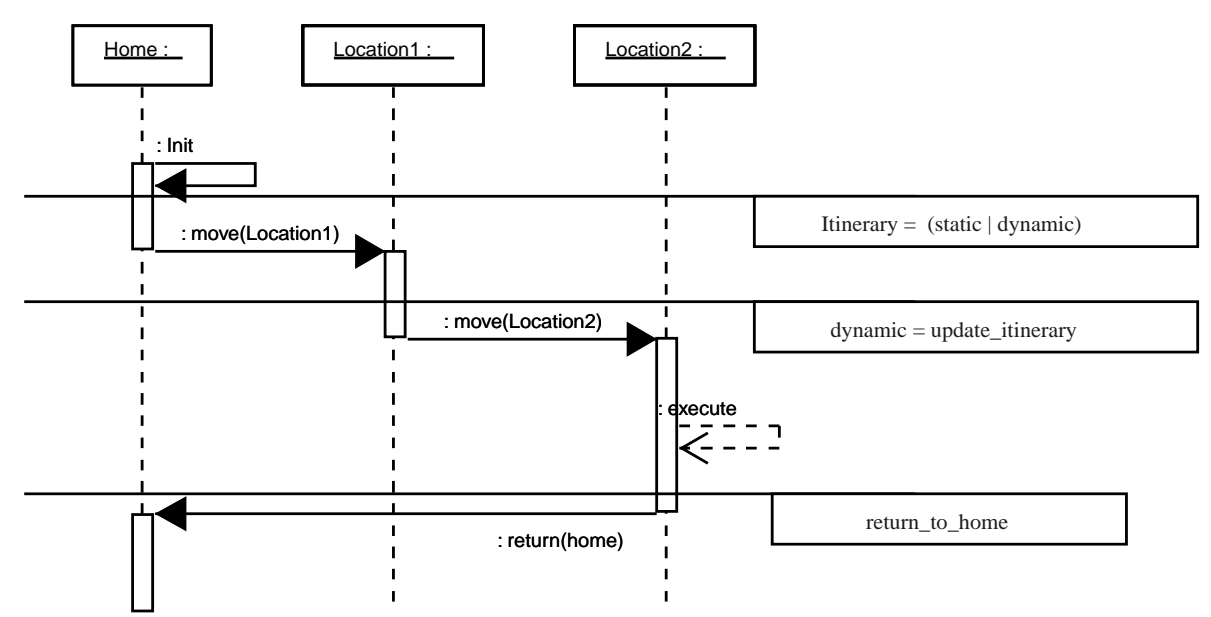

Fig. 9. A Sequence diagram for the Mobile Agent/Itinerary pattern: if itinerary is "dynamic" the user will be able to change it using our existing operators. If itinerary is "static", then an existing topological structure may be used, or one created by the user.

2. Grouping: Operators to support grouping allow patterns to be combined, enabling common operations to be performed on all patterns within a group. Grouping is also useful to support embedding patterns within each other, thereby providing support for hierarchy. These operators may be behavioural or structural.

3. Inquiry: Inquiry operators support comparison between pattern templates, to check for consistency or compatibility (for instance). Inquiry operators may also be used to verify structural or behavioural properties associated with a template, and return a boolean value on evaluation.

4. Ownership: Ownership operators enable the modification and access rights of a template to be controlled. The owner of a template may delegate access to a single user or group of users to modify the template. Similarly, templates within a group may have different ownership, requiring control of access rights to the group. These are primarily behavioural operators.

5. Execution: Operators to support execution provide the mapping between a Problem Solving Environment, and a resource management system, and provide two core functionalities: (1) managing execution of a pattern instance, (2) managing behavioural properties of pattern instances dynamically. These operators may connect to pre-defined scripts for starting, stopping, resuming etc, component execution, or may be mapped to the protocol between a "Super-Scheduler" and local Schedulers necessary to reserve and allocate resources in the Grid - being developed by the GRAAP and Scheduling group within the Global Grid Forum [5]. The execution operators are constrained by the functionality available within a resource management system, and depend on obtaining monitoring information from such systems also. The mapping between the operators and the particular functionality of the resource management system therefore cannot be pre-defined. We therefore rely on an intermediate API (such the Super-Scheduler mentioned above), to enable our operators to be mapped to this API.

Each pattern operator takes a pattern object as input, and returns a pattern, a PT or a boolean result.

\subsection{Structuring and grouping operators}

These operators are used to modify the structural (mainly topological) PTs, maintaining the structural constraints of the original PT, and include:

$\operatorname{Rename}(\mathbf{P 1}, \mathbf{P 2})$ A pattern $\mathrm{P} 1$ is renamed to pattern $\mathrm{P} 2$. This is a structural transformation, and the original structural constraints are preserved. Hence, the constraint when applying this pattern is that both $\mathrm{P} 1$ and $\mathrm{P} 2$ must be a member of the PT class.

Replace(P1, P2) Replace P1, as a single entity, with pattern $\mathrm{P} 2$.

Increase $(\mathbf{P}, \mathbf{n})$ The number of elements in a pattern is increased.

$\operatorname{Decrease}(\mathbf{P}, \mathbf{n})$ 'N' elements are removed from the pattern. 
$\operatorname{Extend}(\mathbf{P}$, element) An element is added to a pattern and its structure is augmented.

$\operatorname{Reduce}(\mathbf{P}$, element) An element is removed from a pattern and its structure is reduced.

$\operatorname{Replicate}(\mathbf{P}, \mathbf{n})$ The component "P" is replicated "n" times, and these replicas are unrelated.

$\operatorname{Embed}(\mathbf{P 1}, \mathbf{P 2})$ Includes a pattern P1 into a higherlevel pattern $\mathrm{P} 2$. The concept of hierarchy is supported here by enabling component place holders to contain other PTs.

Group/Aggregate(P1, . , Pn) A group of "n" patterns is seen as a single pattern, and behave as a single entity.

\subsection{Inquiry operators}

Inquiry Operators return a boolean result and include:

IsEqual(P1, P2) Verifies if two patterns have the same structure.

IsRecursive( $(\mathbf{P})$ Identifies if a pattern is recursive.

IsDisjoint(P1, P2) Identifies if the intersection between two patterns is null. The semantics of what constitutes a particular or exact match is left to the implementation of this operator.

IsSubset(P1, P2) Verifies if a pattern P1 is a substructure of another pattern P2.

IsSuperset(P1, P2) Verifies if a pattern contains a subpattern which matches $\mathrm{P} 2$.

IsComposite $(\mathbf{P})$ Verifies if a pattern is an aggregation, i.e. although "P" may be a group of other patterns it can be manipulated as a single pattern. The operation "IsComposite" returns true if applied to a pattern built with the "Aggregate" operation.

IsInComposite $(\mathbf{P 1}, \mathbf{P})$ Verifies if pattern "P1" belongs to group "P". This operator uses existing pattern templates to perform the comparison.

IsCompatible(P1, P2) Verifies if a pattern is compatible with another one. This operator is used to determine if two patterns are functionally identical. This analysis is undertaken in stages. The first involves checking if two patterns are structurally similar, the second involves checking if the control and data flows between components within a pattern are similar, and the final check involves verifying if all components (or types) within two patterns are identical. All three checks must be valid for the compatibility test to pass.

IsOwner(P1, A) Used to confirm if user/group " $\mathrm{A}$ " is the owner of pattern P1.

\subsection{Ownership operators}

These operators are used to control how a single user or a group of users is allowed to modify a pattern, and include:

Owner $(\mathbf{P 1}, \mathbf{A})$ Used to make user or group "A" the owner of pattern P1.

OwnerGroup $(\mathbf{P 1},\{\mathbf{A 1}, \ldots, \mathbf{A n}\})$ Used to allow all members of a group to own a pattern. All owners have modification rights to the pattern. $A_{i}$ represents a group member.

AssignActivity(P1, Activity $\},$ A) Enables pattern P1 to be modified according to the set "Activity", by owner A. Activity identifies operations that may be performed on a particular pattern, and may be general operators such as write, read, etc, or more complex user defined operations that are bound to a particular object implementation.

RemoveActivity(P1, Activity, A) Enables a single or set of activities to be disabled for pattern P1 and user A.

\subsection{Execution operators}

Execution operators relate to execution scripts on the particular resource management system being used (such as Globus [10]). The types of operators being supported within this category are constrained by the operations being supported within the resource management system, and therefore not all may be usable:

$\operatorname{Start}(\mathbf{P})$ Used to start a pattern's execution.

Terminate(P) Used to terminate a pattern's execution.

$\operatorname{Stop}(\mathbf{P})$ Used to pause a pattern's execution - with the side-effect of checkpointing the state of the execution. Not all resource management systems may support state checkpointing.

$\log (\mathbf{P})$ Used to $\log$ the execution state of a pattern. For this operation, a monitoring service is assumed within the resource management system.

$\operatorname{Resume}(\mathbf{P}, \mathbf{p t})$ Pattern execution is resumed from a previous logged point "pt" (where "pt" may also be chosen to start execution from the beginning).

$\operatorname{Restart}(\delta T, \mathbf{P})$ Repeat execution every $\delta T$ time (periodic execution). Particularly useful for periodic re-starts of an application.

$\operatorname{Limit}(\delta T, \mathbf{P})$ Limits the execution of pattern "P" to a period equal to $\delta T$. If $\delta T$ expires, the pattern is stopped.

$\operatorname{Repeat}(\mathbf{n}, \mathbf{P})$ The execution of pattern "P" is repeated " $n$ " times. 
Steer $(\{$ parameters $\}, \mathbf{P})$ Change the set of " $\{$ parameters $\}$ " associated with a pattern $P$.

ChangeDependencies $(\mathbf{P 1}, \ldots, \mathbf{P n})$ These type of operations allow the execution environment or a user to change the connection(s) between a set of patterns. These operators have a direct impact on how execution of components within a pattern takes place, and therefore need to interface to existing resource management and scheduling systems.

- Synchronise(syncRule, P1, ..., Pn): Change the time dependencies between a set of patterns P1..Pn, according to a synchronisation rule "syncRule" (e.g. all patterns have to produce their results in a synchronous fashion).

- ChangeDataFlow(rule, P1, ..., Pn): Change the data flow connecting a set of patterns, according to a "rule" (such as reverse the direction of a data flow in a pipeline PT). The data flow can specify both the direction of flow and the associated data types.

- ChangeControlFlow(rule, P1, .., Pn): Change the control flow (e.g. switch from a push to a pull strategy), according to "rule".

- ChangeSharedDataDependencies(rule, P1, ..., Pn): Change the way the set of patterns access a shared resource (e.g. switch from exclusive access to multiple entities).

Coordinate $(\mathbf{P}$, rule) Apply coordination rule "rule" to pattern "P" (the rule may be constructed as a sequence of "Execution Control Operations" like start/stop, and "Reconfiguration Operations" like ChangeDependencies).

Combining these operators can lead to powerful execution sequences - such as combining the Steer operator with the Coordinate operator to control how parameter steering is to be supported based on a particular context or data rate (supported through a rule). Each rule can be defined using the deftemplate-defrule structure found in the Java Expert System Shell (JESS) [27]. The use of the behavioural operators Restart and Limit enables a pattern to be run periodically, or be restarted after a particular time interval.

Based on these descriptions, we can classify our design patterns and operators as outlined in Table 1.

Next section describes the semantics of the structural and behavioural operators.

\section{Operator semantics}

The semantics of some of the operators are provided to illustrate the concepts. We start with the structural operators and terminate with a description of some of the behavioural operators.

\subsection{Semantics of structural operators}

Not all structural operators are applicable to all structural patterns (Table 2), and the semantics of each operator may vary with the structural patterns. The semantics of the operators Replicate, Replace, Embed, and Group/Aggregate are independent of the structural pattern to which they are applied. However, the semantics of applying the operators Increase, Decrease, Extend, Reduce, and Rename, are dependent on the pattern template (PT) to which they are applied.

Both the Replicate and Replace are simple operators, as all PT can be replicated - and each replica will have a different identifier. The identifiers themselves can be changed. Likewise, all PT can be replaced with any other PT. The semantics of the Group/Aggregate operator is also quite simple. All PTs can be aggregated in a group template which represents all its members. For example, after grouping a proxy and a pipeline PT into a group, it is possible to subsequently replicate the group PT.

The semantics of the Embed operator defines that the embedded pattern becomes one of the elements of the destination pattern. For example, when embedding a Star into a Pipeline, one of the Pipeline's components will be annotated as having the topological structure of a Star. This specific embedding operation is useful when combining different subsystems in a Grid environment. The user may start by defining a set of Grid services and tools organised in a pipeline. For example, a scientific application (head of the pipeline) generates results for a data analysis tool, which in turn produces data to a visualisation tool (corresponding to the last stage of the pipeline). For instance, users may be familiar with the structure of a problem they are trying to solve, and use the star topology to model a central manager - perhaps a parallel machine or high end server, and a number of sub-servers that interact with it. Assuming that the behaviour of that sub-system follows the Master/Slave pattern, that behaviour can be developed over the star topology.

Hence, the user defines a new star PT (with an adequate number of satellites for supporting the slaves), and embeds this PT in the first position of the pipeline 
Table 1

Pattern templates and operator summary

\begin{tabular}{lll}
\hline & Patterns & Operators \\
\hline \multirow{2}{*}{ Structural } & Pipeline, Star, & Rename, Replace, Increase, \\
& Ring, Bus & Decrease, Extend, Reduce, \\
& Adapter,Proxy, Facade & Replicate, Embed, Group/Aggregate \\
& Master-Slave, Streaming, & IsEqual, IsRecursive, \\
& Client-Server, Peer-2-Peer, & IsDisjoint, IsSubset, IsSuperset, \\
& Mobile Agents/Itinerary, & IsComposite, IsInComposite, IsCompatible, \\
Remote Evaluation, & IsOwner, Owner, OwnerGroup, \\
& Code-on-Demand, Contract, & AssignActivity, RemoveActivity, \\
& Observer/Subscribe-Publish,, & Start, Terminate, Stop, Log, Resume, \\
& Parameter Sweep & Restart, Limit, Repeat, Steer, \\
& & ChangeDependencies.Synchronise, \\
& ChangeDependencies.ChangeDataFlow, \\
& ChangeDependencies.ChangeControlFlow, \\
& ChangeDependencies.ChangeSharedDataDependencies \\
\hline
\end{tabular}

Table 2

Applicability of structural operators over topological and Non-topological structural patterns

\begin{tabular}{|c|c|c|}
\hline Structural Operators & Topological Patterns & Non-Topological Patterns \\
\hline $\begin{array}{l}\text { Replicate, Replace, } \\
\text { Embed, Group/Aggregate }\end{array}$ & Applicable to all & Applicable to all \\
\hline Increase, Decrease & Applicable to all & $\begin{array}{l}\text { Non-applicable to } \\
\text { the Adapter pattern }\end{array}$ \\
\hline $\begin{array}{l}\text { Extend, Reduce } \\
\text { Rename: }\end{array}$ & Non-applicable & Applicable to all \\
\hline $\begin{array}{l}\text { - to restructure a pattern into } \\
\text { a topological pattern }\end{array}$ & Applicable to all & Applicable to all \\
\hline $\begin{array}{l}\text { - to restructure a pattern into } \\
\text { a non-topological pattern }\end{array}$ & $\begin{array}{l}\text { Depends on the cardinality } \\
\text { of the pattern templates }\end{array}$ & $\begin{array}{l}\text { Depends on the cardinality } \\
\text { of the pattern templates }\end{array}$ \\
\hline
\end{tabular}

PT, thus producing a hierarchical structure (see Fig. 10). Similarly, the embedding of a Facade into a Proxy may result in the subject component in the Proxy pattern being annotated as being a Facade.

The Increase and Decrease operators can be applied to all selected structural patterns, except the Adapter pattern (Table 2).

When applied to the Pipeline and Ring patterns, the Increase operator increases the number of elements in the structure (e.g. it is possible to increase a two element pipeline to a four element pipeline - see Fig. 11). Over the Star pattern, the operator increases the number of satellites in the structure. Similarly, the $D e$ crease operator reduces the number of elements in the structure over which it is applied. Both Increase and Decrease operators may be applied to the Facade pattern, resulting in the increase/decrease of the number of subsystem classes (see Fig. 6). The same operations over the Proxy pattern result in the increase/decrease, respectively, of the proxy elements in the pattern (see Fig. 11).

For example, the proxy pattern may be extended by adding a proxy component to an existing proxy (see Fig. 12). This situation occurs in mobile agent/object systems, where the sequence of proxies is used for locating the agent/object (via a chain for message forwarders, for instance). The message forwarding mechanism is also useful when implementing authentication requests via a "security chain" - whereby each node forwards requests for authentication to another node along the path. Likewise, the Facade pattern may be extended by adding a new facade component which "hides" an existing facade component, which in turn becomes a simple subsystem class for the new facade component (see Fig. 12).

For the Rename operator, the cardinality of the patterns being renamed may be important in determining whether the operator can or cannot be applied (see Table 2). For instance, any topological pattern may be renamed into any other topological pattern, independently of the cardinality of the pattern. For example, a Pipeline pattern can be transformed into a Ring pattern, by connecting the first and last components of the Pipeline (see Fig. 13). Similarly, a Pipeline can be transformed into a Star, by taking one of the Pipeline's components as the nucleus of the Star pattern, with the other components becoming satellites. Similarly, any non-topological pattern can be restructured into a topo- 


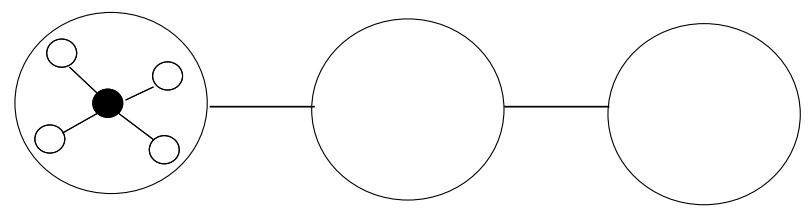

Fig. 10. An example of a pipeline template with an embedded pattern (a star) in the leftmost component.

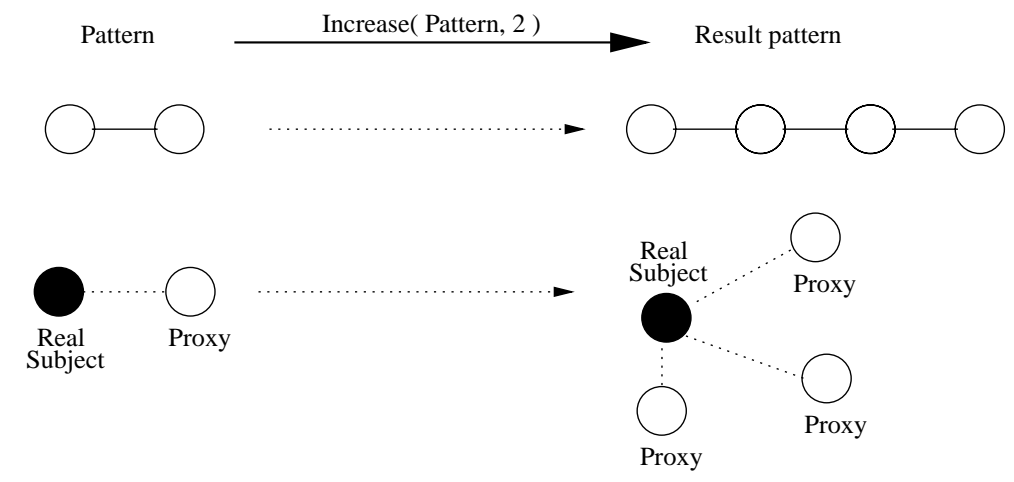

Fig. 11. The Extend and Reduce operators can be applied to the selected design patterns but not to the topological patterns.

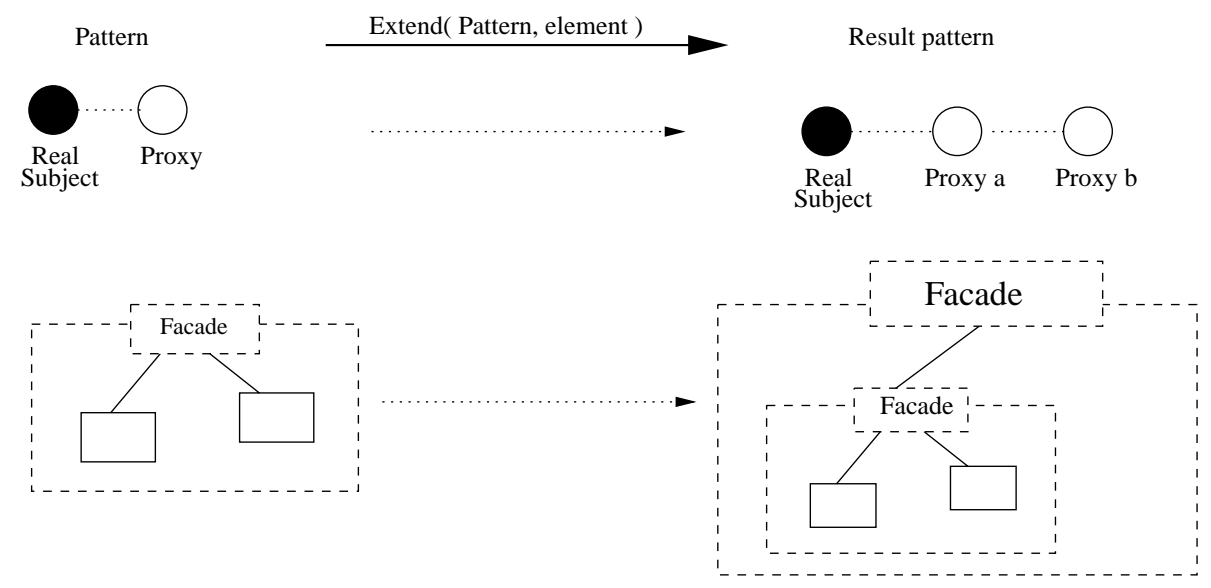

Fig. 12. Examples of the Extend operator over the Proxy and Facade Pattern Templates.

logical pattern, as long as the cardinality of the original pattern is maintained. For example, in Fig. 13, a Proxy pattern template containing three proxies is renamed into a star, which will have three satellites. These structural operators therefore transform one pattern to another one within the same class. Additional transformation may be undertaken by a user directly using an editor - although this does not provide any checking that the transformation will leave a pattern class invariant. Also, when using the Rename operator, the cardinality of the pattern template must be preserved. For example, it is not possible to rename a pipeline pattern with five elements into an Adapter, because their car- dinality is different. However, the same pipeline can be renamed into a facade by annotating one of the elements of the pipeline as the facade component, and the other elements as the sub-system classes (see Fig. 13).

\subsection{Structural operators in use}

This section gives some examples of the application of structural operators in the context of the application introduced in section 2. Figures 14 and 15 describe a possible sequence of steps to build the PSE configuration shown in Fig. 1, according to the patterns identified in Figs 7 and 8. 

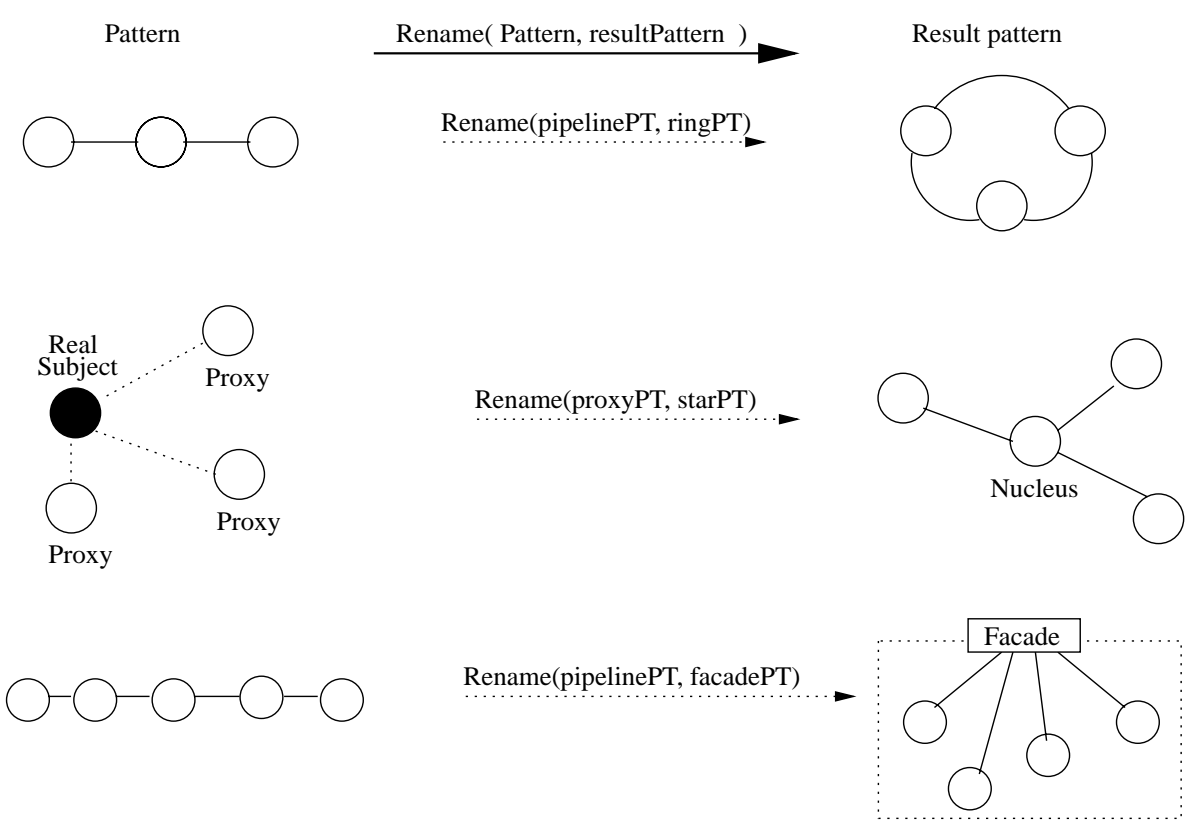

Fig. 13. Examples of the Rename operator over a Pipeline and a Proxy Pattern Templates.

STEP 1:

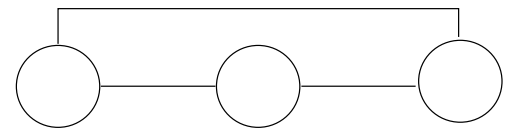

STEP 2:

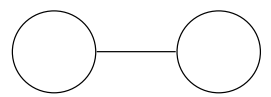

STEP3:

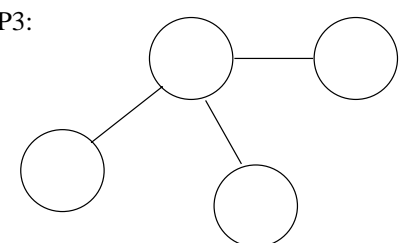

STEP 4

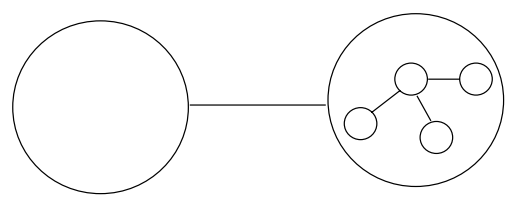

Step 1 - Creation of a ring PT with three component place holders (for the problem solver, the monitoring service, and the steering interface).

Step 2 - Creation of a pipeline PT with two component place holders (for the problem solver and the database system).

Step 3 - Creation of a star PT for the database system (the frontend will be the nucleus and the slaves will be the satellites).

Step 4 - Embedding of the star PT into the pipeline PT built in step 2.

Fig. 14. Initial steps for building the PSE depicted in Fig. 1.

In step 1 (Fig. 14), the user creates a ring pattern template (PT) with three place holders to represent the components connecting the Problem Solver, the Monitoring service, and the Steering Interface. Next, the user creates a pipeline PT with two component place holders to represent the connection between the Problem Solver and the Database System (step 2). This pipeline will be embedded in the first component place holder of the ring, but first the user must create a PT to represent the Database System. In step 3, the user creates a star PT with three satellites that will be instantiated to the Database sub-systems. In step 4, the user applies the Embed structural operator over the pipeline PT with the star PT as argument, to be embedded in the second component place holder of the pipeline PT.

In step 5 (Fig. 15) the user applies the Embed struc- 


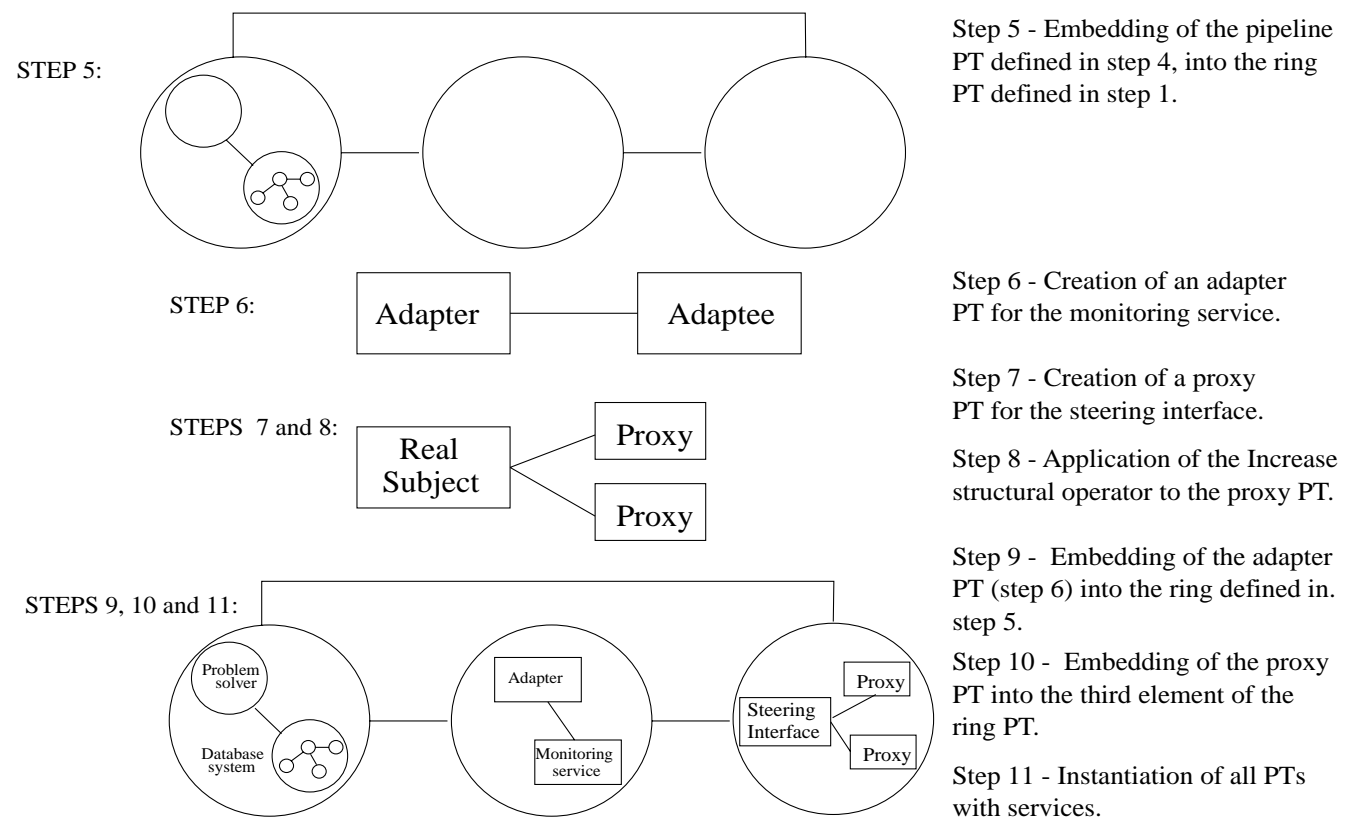

Fig. 15. Final steps for building the PSE depicted in Fig. 1.

tural pattern to include the pipeline PT obtained in step 4 into the first component place holder of the ring PT (previously defined in step 1). Next, the user creates an Adapter PT template to represent the Monitoring service (step 6). In steps 7 and 8, the user creates the structure for the Steering Interface (which will be accessed by other users). To achieve this, the user creates a Proxy PT and then its proxy elements are increased by one through the application of the Increase structural operator. In steps 9 and 10, the user embeds the Adapter PT and the Proxy PT in the ring's second and third component place holders, respectively. Finally, in step 11, the user instantiates all pattern templates with the selected services. The user may now apply the appropriate Behavioural Patterns (e.g. as defined in section 3.5), and run the application using the Behavioural Operators to control its execution.

As a final remark, the user may eventually apply the structural operators again to reconfigure the application. For example, to perform a post-mortem analysis of the data produced by the Problem Solver as illustrated in Fig. 4 (see section 2), the user may apply the Rename structural operator to transform the ring into a pipeline, and define the Database system as the first element of the pipeline.

\subsection{Semantics of behavioural operators}

In this sub-section we focus on the semantics of the Execution Operators which act upon pattern instances.
Pattern instances are structural pattern templates which have been assigned a behaviour pattern, and within which components have been already bound to executable component instances.

The Execution Operators can be divided into two groups. The first group controls the execution of a pattern instance, and includes: Start, Terminate, Stop, Log, Resume, Repeat, Restart, Limit, and Steer. As previously mentioned, the applicability of these operators depends on the kind of runtime environment available. For instance, the Globus system may support particular execution mechanisms that are not supported in Legion, etc. For now, we assume the existence of at least the following operations - and the ability to communicate these requests to the underlying runtime system: to start running the component instance; to suspend/stop the current execution by temporarily saving the execution status; to resume the execution from the saved status; and to terminate the execution of the component instance. The second group of Execution Operators allows changes to the coordination and reconfiguration of pattern instances, and includes: ChangeDependencies and Coordinate. Currently, we limit these changes to the pattern interactions. The examples that follow are restricted to the first group of execution operators.

For illustration purposes we describe the semantics of the execution operators using a synchronous model, namely the CO_OPN/2 formalism [30,31]. For instance, we assume that the operators Start, Terminate, 
and Stop act synchronously over all component instances contained within a pattern. Hence, the invocation of the Start operator would imply invoking, simultaneously, the start operation on all component instances. It is also possible to consider cases where the invocation of components is asynchronous - however, we restrict our analysis for now for the synchronous case - primarily because of the particular benefit the $C O \_O P N / 2$ tool we use offers in undertaking such an analysis.

Figure 16 gives an application example of the Start and Terminate operators over a particular instance of a pattern. The notation used is adapted from the CO_OPN/2 formalism [30], which provides ObjectOriented abstractions for modelling systems, and where synchronisation between object invocations can be modelled. Moreover, the CO_OPN/2 formalism allows the functionality of each object to be represented as a Petri-Net - where data flow in the Petri-Net can model abstract data types. The objects in CO_OPN/2 are seen by us as components. Figure 16 shows an example of a pipeline instance with three components, where each component is represented as an ellipse. As we are not, at this stage, concerned with the internal behaviour of components, we do not show Petri-Net blocks for these. The input ports (method calls) available on a component are represented by black rectangles along the border of the ellipse. Similarly, the output ports (or Gates) are represented as white rectangles. Synchronisation between components is achieved if there are arrow transitions connecting the component ports. This means that the methods associated with the ports are invoked synchronously. As such, the figure shows that the output port (Output $(d))$ of the leftmost component in the pipeline instance is synchronised with one of the input ports $(\operatorname{Input}(d))$ of the middle component. As soon as the Output method is invoked, the Input method in the other component is invoked as well, and data is exchanged in the process. Likewise, the output port (Output $(d))$ of the middle component is synchronised with the port $\operatorname{Input}(d)$ of the rightmost component. In this way we represent a simplified kind of Producer/Consumer behaviour between the components of the pipeline.

Another important aspect of modelling with CO_OP $\mathrm{N} / 2$ is the notion of a context - which in the figure is represented as a rectangle with round corners. A context is an entity encapsulating a set of components and the coordination rules that constrain those components. As with components, contexts also have input and output ports. Using the CO_OPN/2 formalism, we represent context's ports as bi-coloured rectangles. In the inport ports, the dark part of the rectangle is on the outside of the context and the white part is on the inside, and in the output ports is the opposite. In Fig. 16 there is a single context which represents a pipeline instance encapsulating three components. The invocation of the Start method over the pipeline context implies the simultaneous invocation of the Start method of every component. We represent this simultaneous invocation by the simultaneity symbol // which belongs to the CO_OPN/2 formalism. The simultaneity symbol is one of the synchronisation policies that the formalism provides. The other two policies are the sequence (the methods are invoked in sequential order) and the alternative or nondeterminism (the method to be executed is selected in a non-deterministic way among a set of available alternatives).

The Terminate operator has a similar behaviour to the Start operator, as shown in Fig. 16. The invocation of Terminate over the pipeline implies the simultaneous invocation of the Terminate method at all component instances.

The semantics of the Stop operator implies the immediate suspension of the execution of all component instances - hence it is similar to the Terminate operator. Using this operator, however, also causes the state of all component instances to be recorded. Figure 16 could be extended to represent the Stop operator by including an extra component in the pipeline context. This extra component would be responsible for collecting, simultaneously, the execution state of all components - prior to terminating the execution of a component.

The semantics of the Resume and Log operators are related. While the latter implies saving the execution state at well identifiable points, the former is used to continue the execution of the pattern from one of several points identified by the $\log$ operator. Figure 17 represents an example of the Log operator for storing data flowing in the pipeline instance of Fig. 16. In the example, we assume that the Log operator is invoked at the same time as the Start operator, and that the logging operations are realised by the Log component instance. The figure shows a Petri-Net representing a possible behaviour for the component supporting the Log functionality. The component used for logging data must be started simultaneously with the execution of other components - hence the Init Jogging method call. Subsequently, whenever an output is generated by a pipeline stage, the Log_data method is invoked, causing data to be stored into a Repository. The identity of the component which generated the output in the pipeline is 

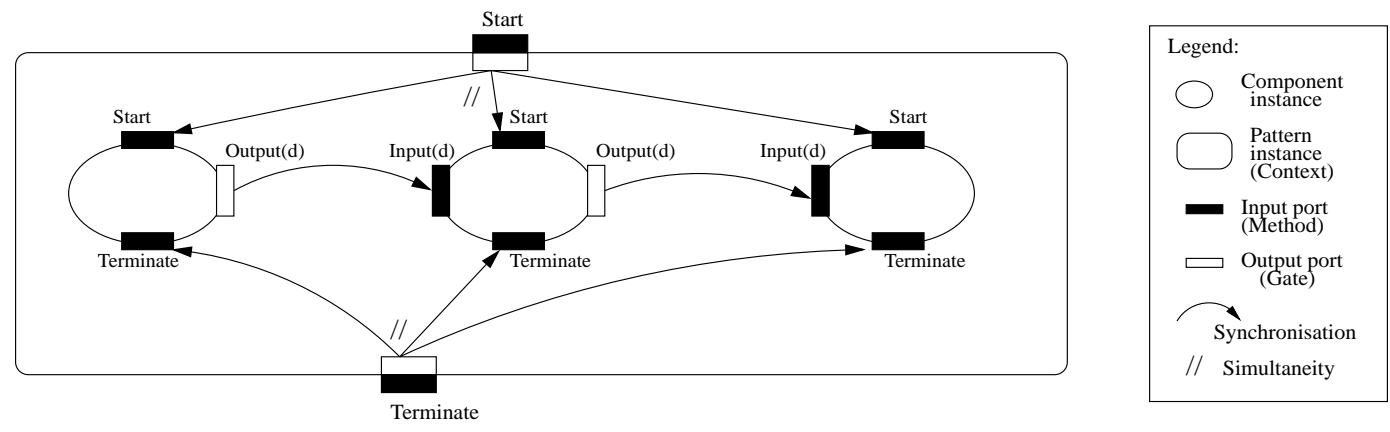

Fig. 16. Example of the Start and Terminate operators over a pipeline pattern instance.

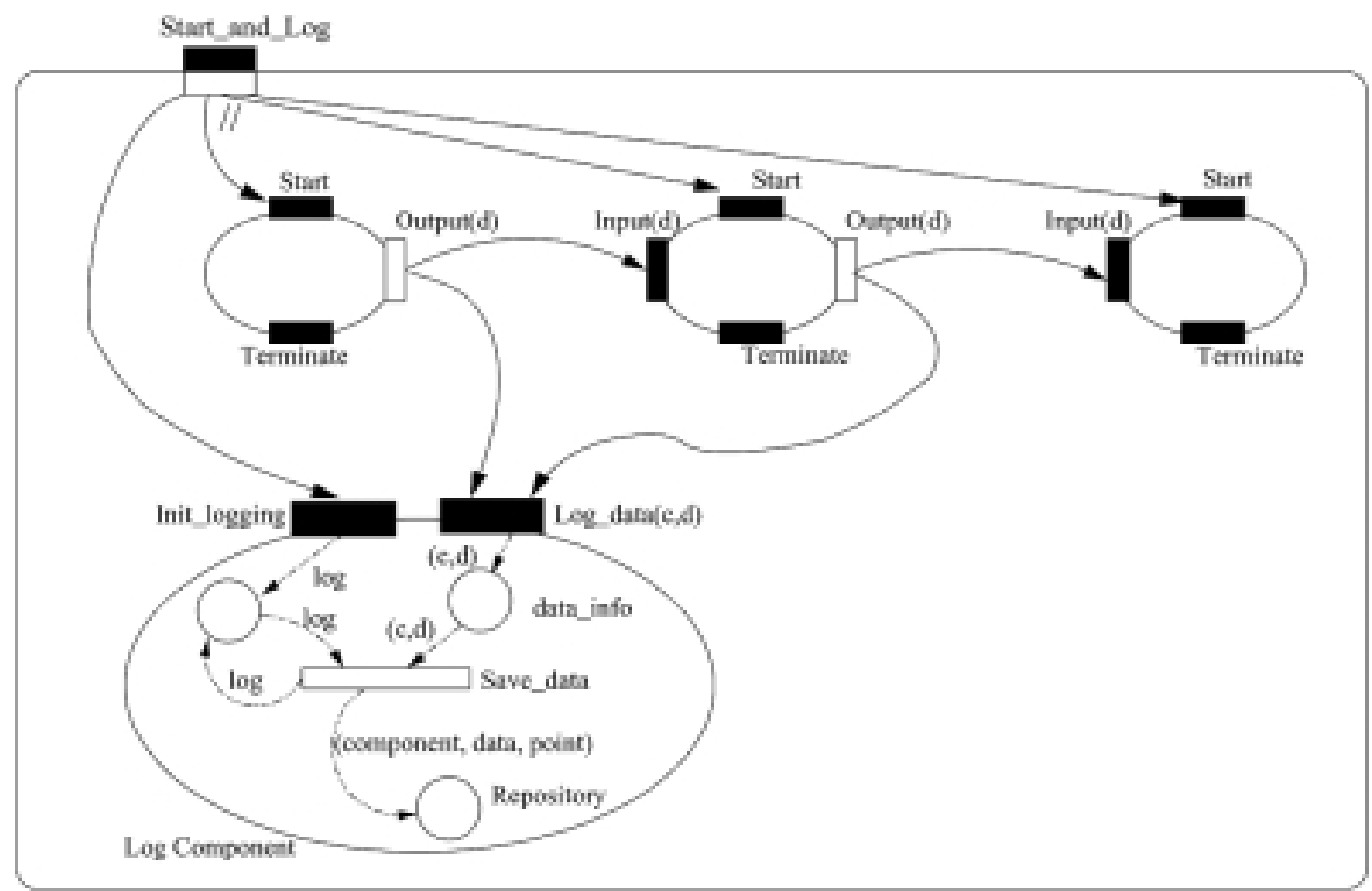

Pipeline context

Fig. 17. Example of the Log operator invoked simultaneously with the Start operator over a pipeline instance.

also recorded via Saved_data (the $c$ parameter in the Log_data method identifies the component).

For the Repeat operator it is necessary to provide a counter to record the number of times a pattern instance has been executed so far. It is also necessary to identify when the execution must terminate. Such an operator is particularly useful for supporting loops in scientific codes. An example of the Repeat operator can be provided by extending Fig. 16 with the following elements: the pipeline context would have a new output port, e.g. end_of_execution, which would be automatically invoked at the end of the pipeline instance's execution; a new context encapsulating the pipeline context would be added; another component would be created in the outmost context that would represent the behaviour of the Repeat operator. This component would implement a counter, initialised with the number of times the pipeline pattern would have to be repeated. The counter would be decremented synchronously with the invocation of the output port "end sf execution".

The Restart and Limit operators are similar in the sense that both depend on the notion of time for controlling a pattern. The Limit operator waits until the time value received as input expires - followed by the termination of the pattern instance managed by this operator. The Restart operator waits for the expiration of 
the value held by the input time token, and invokes the start operation over the pattern it manages. Figure 18 gives an example of the semantics of the Restart operator when applied over the pipeline instance of the example in Fig. 16. Figure 18 adds a new context (Restart context) to Fig. 16, which encapsulates two components: one component is a timer which generates a tick at a specific time interval (e.g. a second); the second component represents the steps necessary for the restart operator. One of the transitions in the Petri-Net in this second component decrements the time interval received as argument at each tick of the timer, and keeps the result in place counter. When the counter reaches zero, a second transition is fired which launches the restart of the pipeline's execution and initialises again the place counter with the original time interval (kept in the place time).

\subsection{Behavioural operators in use}

Figures 2 and 3 (section 2) describe a re-configuration example where the Monitoring service is replaced with a more complex service (Monitoring and Statistics Service). Applying the Stop behavioural operator enables the complete workflow to be terminated. Alternatively, the user might also apply the Stop operator over individual patterns - such as the Proxy pattern instance that represents the Steering interface. The user must then replace the Adapter pattern with an instance of the Monitoring and Statistics Service. Finally, the Restart operator is applied to this new service (and eventually also to the Proxy pattern instance that represents the Steering Interface).

Having illustrated the application of structural and behavioural operators, next section describes the current implementation status.

\section{Implementation Issues}

This section describes the existing implementation of structural patterns and structural operators over the Triana environment. It also points out the continuation of the work on the implementation of behavioural patterns and operators.

\subsection{The triana environment}

Triana [19] is a Java-based workflow environment that supports application construction based on distributed components. Application execution results from the collaboration between various network Peers (see [20]), which act both as clients (for local users' requests) and as servers (for remote peers' execution requests). Locally, each peer may access existing Grid services to execute high-throughput computations.

Through the Graphical User Interface (Fig. 19) provided with Triana, users have access to services/tools (e.g. components for Signal Processing, Mathematical Calculations, etc.) that can be easily composed for building scientific applications. Users drag and drop components from the toolbox onto the scratch pad on the right side, and create a workflow by dragging cables that connect components together. Sender components are connected through output ports (or nodes) on the right-side, to receivers' input ports (on the left-side). Users may also group selected components together into a component which represents the entire set. This "group component" also has input and output ports for connecting the group (and some of its hidden elements) with other components.

In Triana, execution follows the data-flow model: as soon as data arrives to a component's input port, the service it represents is launched. Moreover, users may define which parts of the workflow may be executed remotely. The local Peer makes the necessary requests to remote Peers, collects the results, and displays them through the GUI. Execution may be supported by each Peer itself or may be realised by accessing a local resource manager (e.g. Globus GRAM [13]).

\subsection{Structural patterns and operators in triana}

Structural Patterns are available in Triana's toolbox as normal components (from a graphical perspective). The user just has to drag and drop them into the scratch pad and initialise them. For example, Fig. 19 shows a Ring PT and a Star PT that resulted from the initialisation of DrawRing and DrawStar, respectively. Each pattern template represents a set of component place holders called DummyUnits, which can be instantiated to other PTs or tools from the toolbox. DummyUnits are connected together according to the PT's specific structural Pattern (i.e. ring, star, etc).

Structural Operators are available as parameters to pattern templates. For example, Fig. 20 shows the application of the Embed structural operator to the Ring PT. To use this, the user must invoke the Ring's PT parameter window to specify that the Pipeline PT should be embedded into the first Ring PT's place holder (called DummyUnit). The Pipeline PT already has an an embedded Star PT. This example shows one of the 


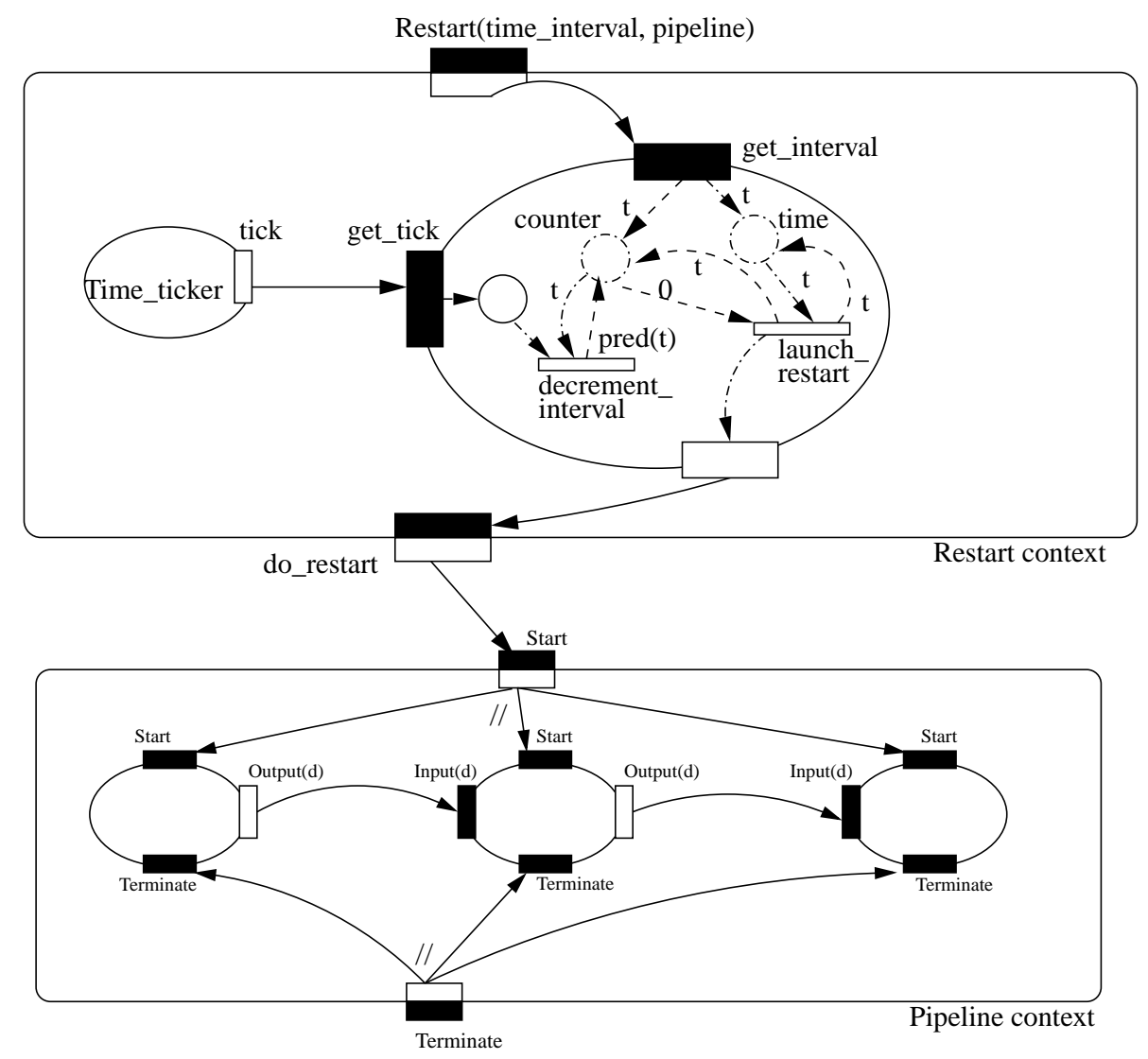

Fig. 18. Example of the Restart operator over a pipeline instance.

steps to build the structure shown in the example described throughout the paper.

Structural pattern templates are implemented as groups in Triana. The group contains: a) the connected DummyUnits, and b) a control task that keeps track of the number of component place holders (and their connections), listens to relevant events (like requests to instantiate DummyUnits), and supports the execution of the structural operators.

The user may now compose an application by combining PTs with existing components, and may save them as a group component in the toolbox for later reuse. Once an application has been constructed - one or more behavioural patterns may be used to specify the data and control flows between components.

\subsection{Usage scenario: Galaxy simulation}

To illustrate the use of PTs in Triana, we utilise a "Galaxy Formation" code example. The example involves generating the position of particles and subsequently animating these - using a combination of
"DataReader" and "Animation" modules from Triana. A data file is loaded by a single Data Reader Unit within Triana, and passed to all the Triana nodes. Nodes then buffer the data for future calculations. Note that the data file could be copied beforehand and distributed in a parallel way also. The loaded data is then separated into frames, distributed amongst the various Triana servers on the available network and processed to calculate the column density using smooth particle hydrodynamics. These types of simulations can usually generate large data files containing snapshots of an evolving system. They are therefore quite representative of the types of applications that may be executed over Grid infrastructure. In this particular example, after undertaking a simulation run, a snapshot is produced - and which is independent of others over time. This suggests that any data analysis on frames can be carried out independently. Grid resources are used in this instance to distribute and remotely process data frames, which finally return a small image to the visualisation/controlling client. The images can be subsequently re-assembled 


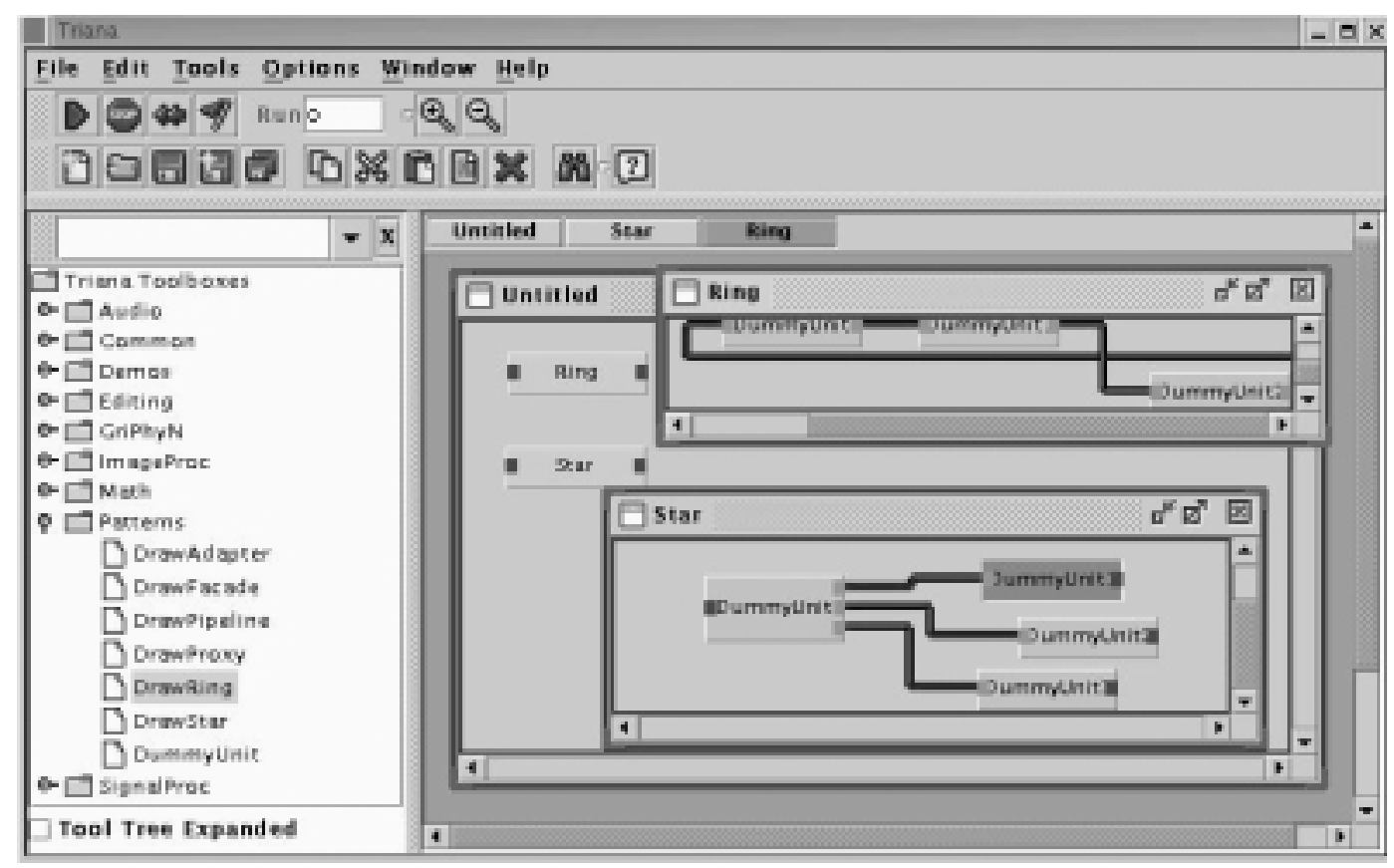

Fig. 19. The Triana Graphical User Interface.

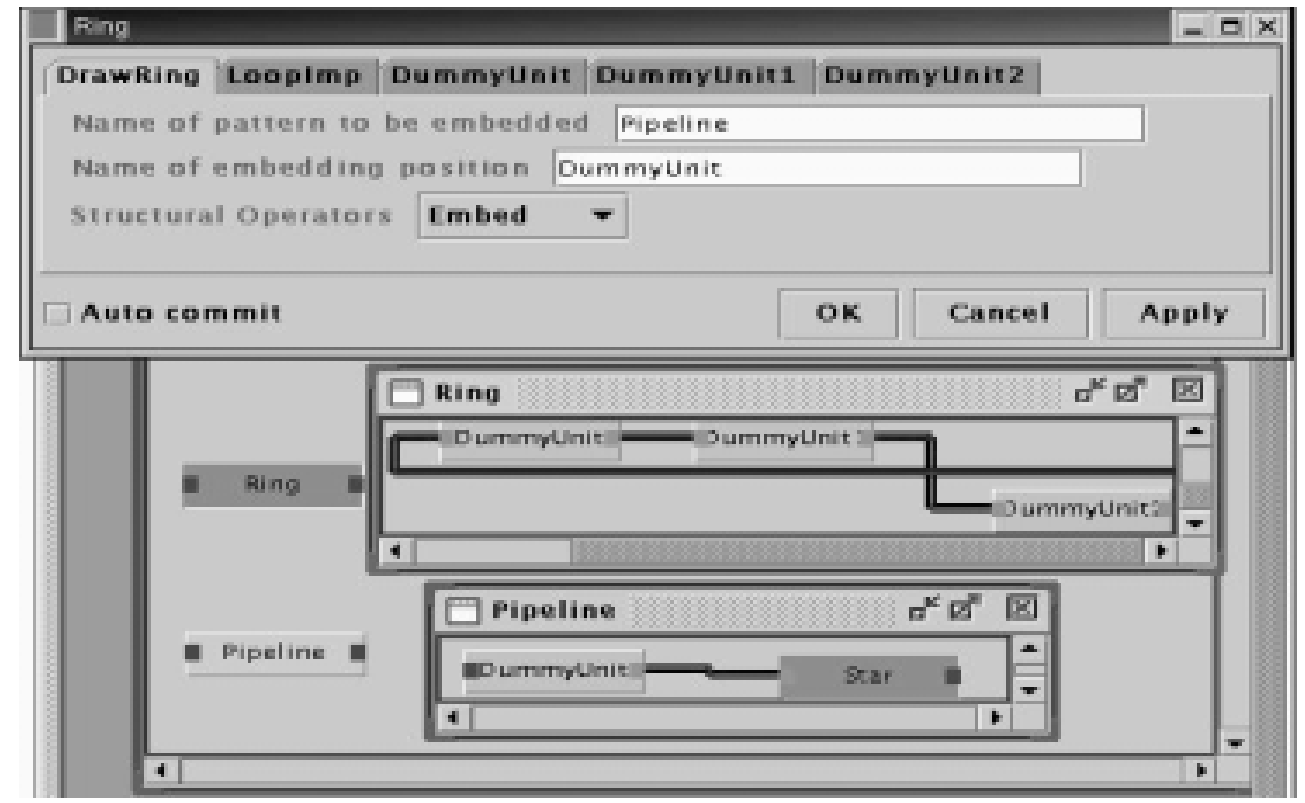

Fig. 20. Application of the Embed Structural Pattern to the Ring Pattern Template.

in real-time into the correct chronological order to generate a smooth animation.

Galaxy and star formation simulation codes generate binary data files that represent a series of particles, along with their associated properties as a snap shot in time. The user of such codes would like to visualise this data as an animation in two dimensions, with the ability to vary the perspective of view, and project that particular two dimensional slice and re-run the animation. Due to the nature of the data, each frame or snap shot is a representation at a particular point in time of the total data set. It is possible to distribute each time 


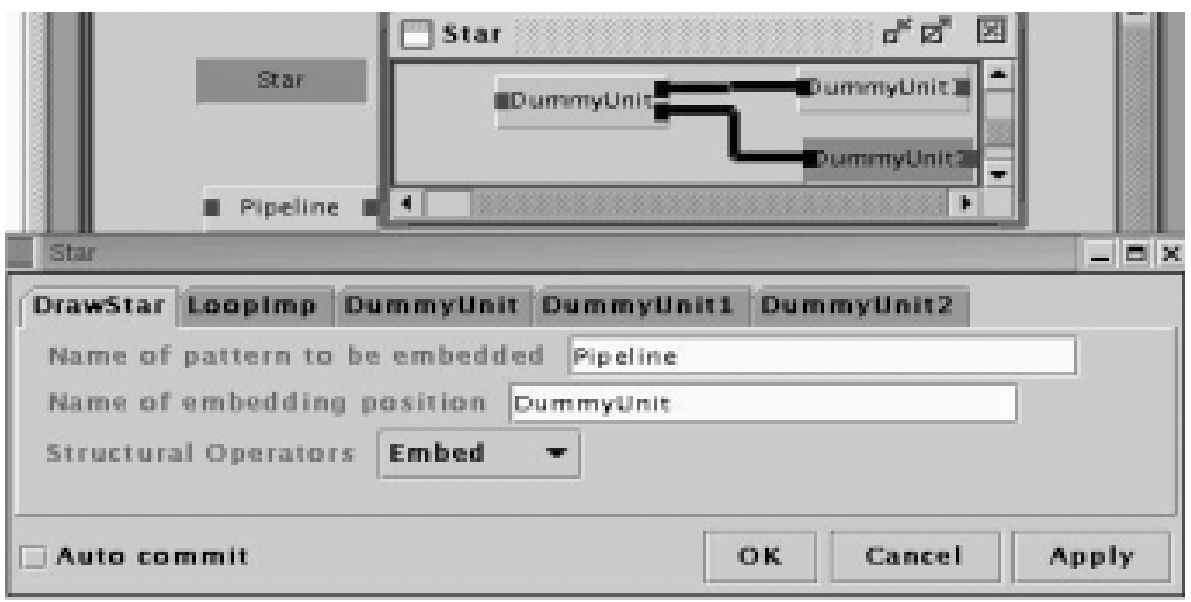

Fig. 21. The animation is supported by a pipeline PT which is embedded in the nucleus of the star PT.

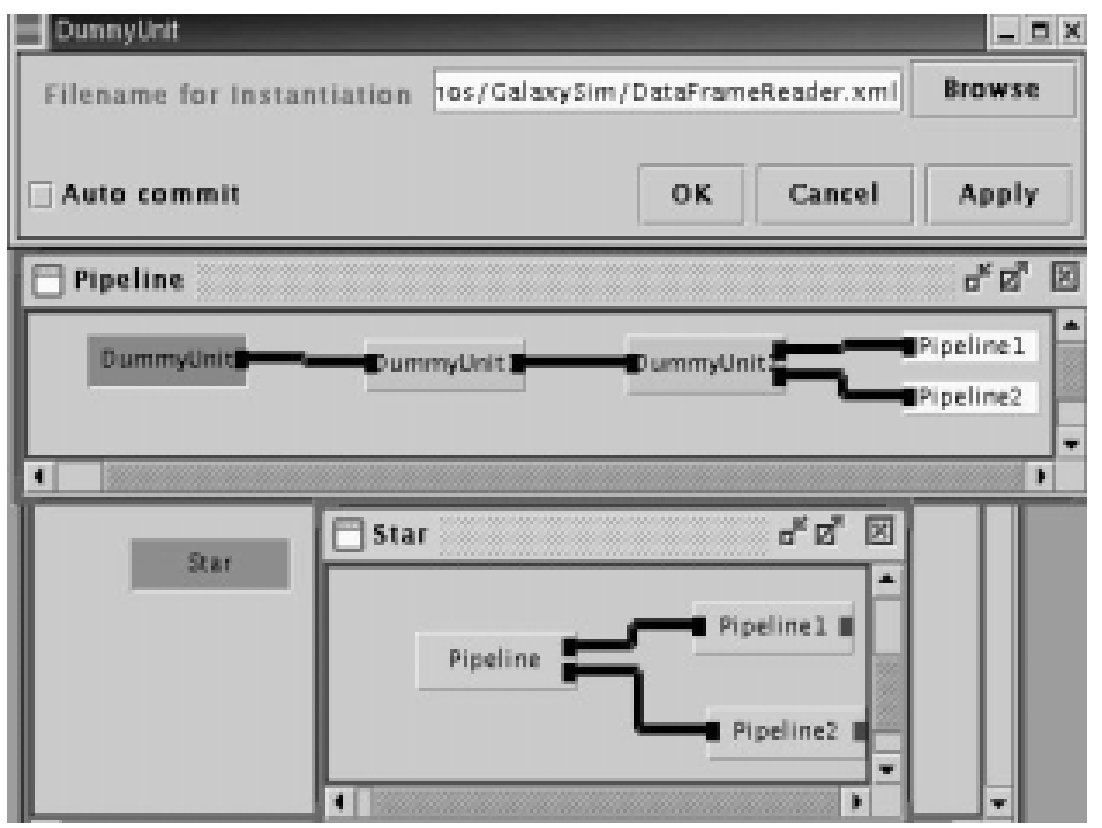

Fig. 22. An example of a component place holder instantiation.

slice or frame over a number of processes and calculate the different views based on the point of view in parallel.

The Galaxy formation example may be represented by a Star PT, where the nucleus contains the actions necessary to generate and control the animation execution, and the satellites represent image processing and analysis actions. In this way, the same animation can be simultaneously analysed/processed in different ways. Figure 21 shows a Star PT with three component place holders - the satellites (DummyUnitl and DummyUnit2), and the nucleus (DummyUnit). As the animation is developed in stages, these are represented by a Pipeline PT. Figure 21 shows the Pipeline PT embedded in the nucleus of the star by selecting the embed structural operator, and by identifying the embedding position (DummyUnit).

Figure 22 shows the Star PT with the embedded Pipeline PTs, to support the image processing activities required to generate the animation. The next step involves instantiating the place holder (named DummyUnit) of the pipeline (in this case a DataFrameReader is selected from the Triana toolbox) - as illustrated in Fig. 22. Figure 23 shows the final configuration, with 


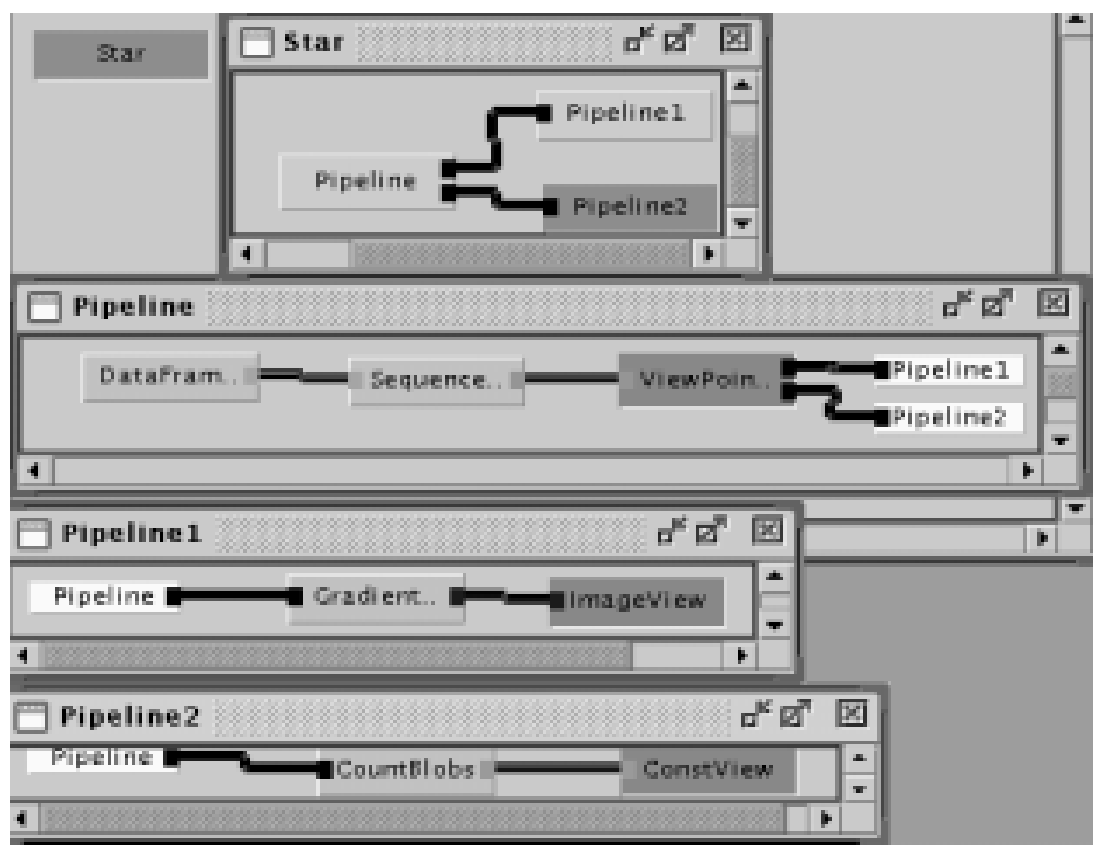

Fig. 23. A possible final configuration for the image processing of the "Galaxy Formation example".

all component place holders instantiated with units. Hence, the binary data file produced by the simulation code is loaded by the DataFrameReader unit. The frames are sent to the SequenceBuffer unit - a media controller that allows the replay of the application. The user may stop the animation, rewind it, restart it, etc. The ViewPointProjection unit takes the 3D data and projects this onto a $2 \mathrm{D}$ space outputting a standard PixelMap. The user may change the point of projection by changing parameters representing the $(\mathrm{x}, \mathrm{y})$ coordinates. The resulting animation images are analysed/processed in parallel in Pipelinel and Pipeline2. The GradientEdge unit selects images based on a gradient edge detector, and subsequently displays these using the ImageView unit. In Pipeline2, the number of non-black objects in each image are counted by CountBlobs unit and displayed in ConstView unit.

Figure 24 shows the output of units ImageView and ConstView, and shows the parameter interface panel for unit SequenceBuffer.

Alternative Configuration: A possible alternative configuration decouples the view point projection of the simulation, from the reading of data, allowing parallel animations with different view points. A Star PT supports the configuration (Fig. 25): the data is read at the nucleus by unit DataFrameReader (the unit SequenceBuffer was omitted for simplification) and sent to the satellites Pipeline PT and Ring PT to be pro- cessed. The DataFrameReader unit may interact with the satellites according to a Streaming behavioural pattern. In the satellite supporting the Pipeline PT (see Fig. 25), a user may select the appropriate viewpoint through the ViewPointProjection unit. The resulting images may be scaled by the ScaleImage unit and subsequently displayed by the ImageView unit. The Producer/Consumer behavioural pattern may represent the interaction between the ScaleImage (the producer) and the ImageView unit.

In the satellite with the Ring PT (Fig. 25), the viewpoint is automatically selected according to the number of non-black objects in each image. For the Pipeline 1 stage contained within the Ring PT (see Fig. 26), the images produced by ViewPointProjection are visualised in the ImageView unit. In the next stage of the ring, the CountBlobs unit counts the number of non-black objects in each image, followed by a stage (Pipelinel) which evaluates if it is necessary to change the view point. If this is the case, the Scroller unit is triggered and inputs the new value to the " $x$ " coordinate parameter for the unit ViewPointProjection, closing the ring in this way.

\subsection{Ongoing work}

The continuation of this work is to extend Triana to support different behavioural patterns. The behavioural 


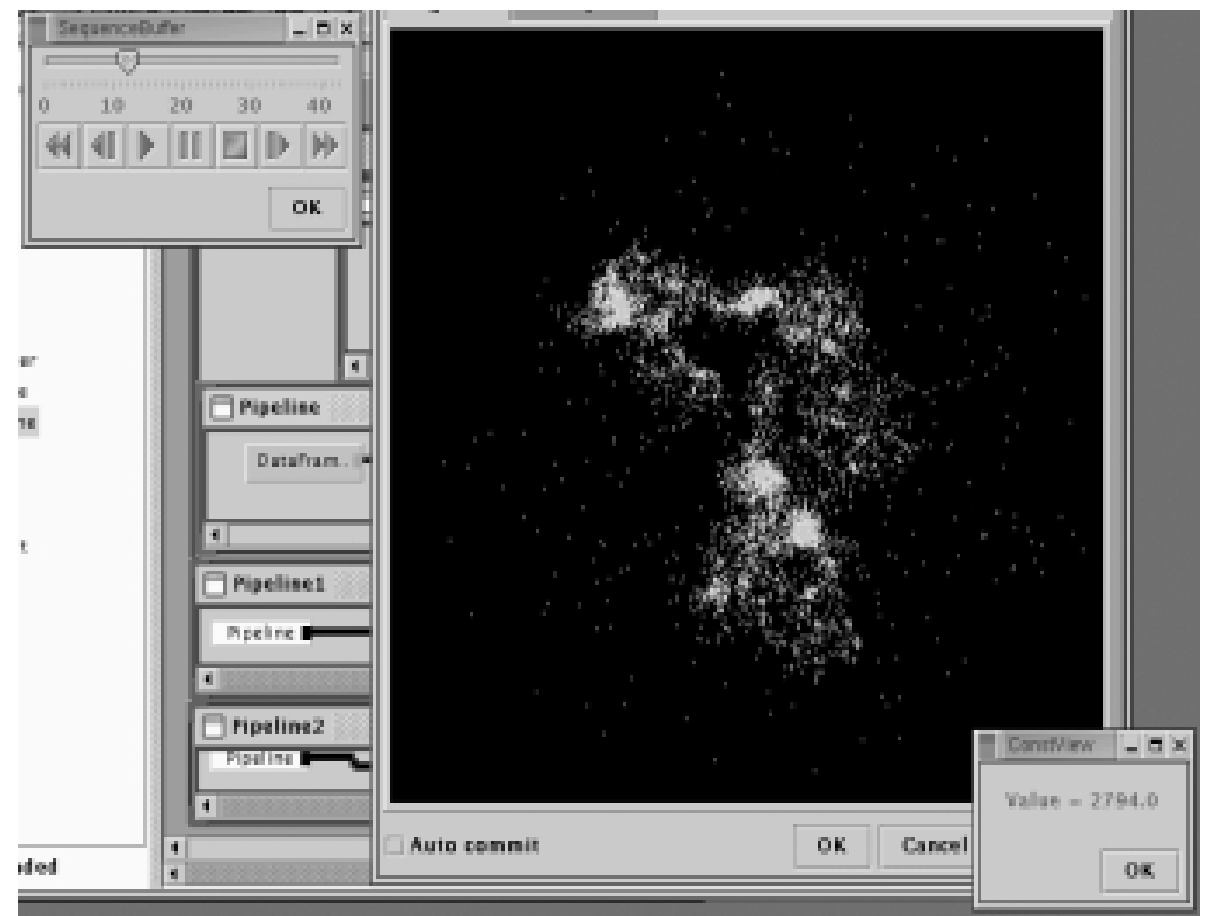

Fig. 24. Execution snapshot for the selected configuration.

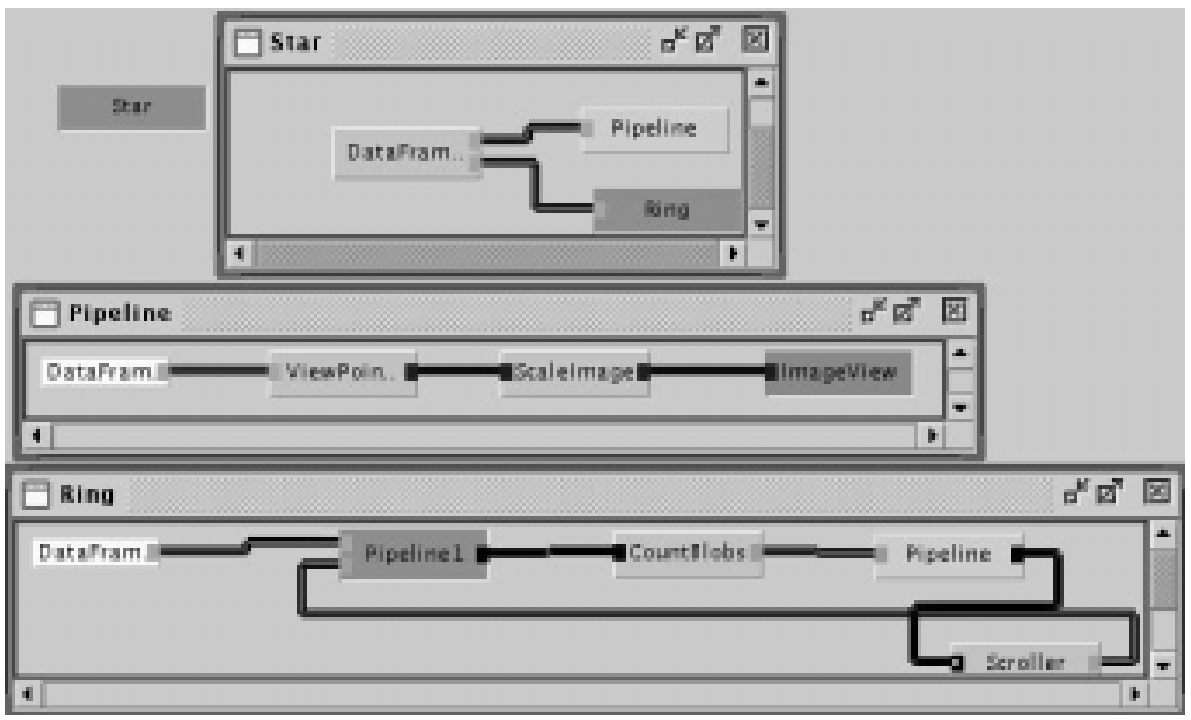

Fig. 25. Parallel animation execution with different view points.

patterns will be stored as Java classes, and will be applied to a pattern template visible on the scratch pad. In case of multiple pattern templates, the particular template should be explicitly identified. In an application composed of both structural PTs and components, the behavioural patterns may only be applied to the struc- tural PTs. This is achieved by having the user annotating a start-point and an end-point on the structural PT, and then running the appropriate behavioural pattern (e.g. selection of the first and second elements in a Ring PT and application the Observer behavioural PT). The implementation of behavioural patterns will require the 


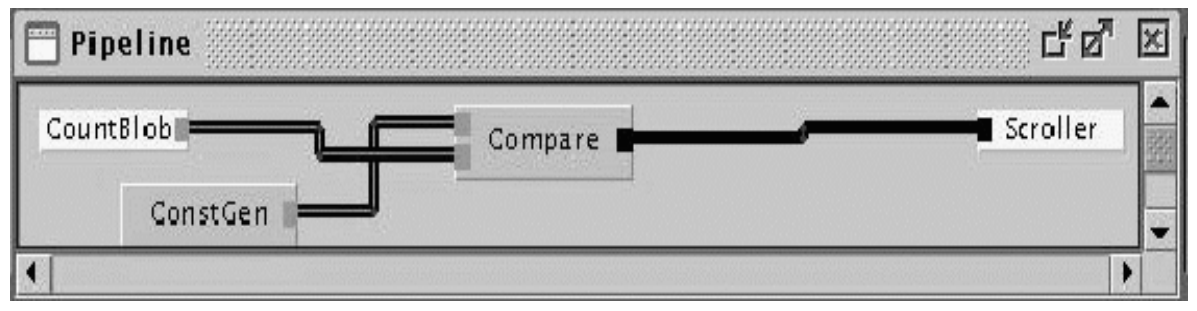

Fig. 26. Detail of the stage named Pipeline in the Ring PT from Fig. 25.

challenging task of supporting the switching between the Triana's dataflow execution model, and more complex control flows. The subsequent step will be the implementation of behavioural operators for execution control and reconfiguration (e.g. management of the flow within the PT).

\section{Conclusion}

This paper outlines an approach for constructing Grid based applications, using a set of Pattern Templates and Operators. Pattern templates are specified in UML, and consist of a collection of class libraries which describe properties associated with each type of pattern. Operators are of two types: structural and behavioural, and enable a developer to modify the template itself, or data/control flows within it, respectively. UML descriptions of common structural patterns are provided, along with the definition and semantics of the operators. The operators are aimed at being used within an existing Problem Solving Environment, and to extend the functionality of the environment. Some transformations between operators also require the PSE to label/annotate elements of the Pattern Template, to enable appropriate associations. These annotations are used to aid operator semantics. The need for software engineering support of this kind arose from our investigation on existing work on Grid computing - which has primarily focused on implementing workflow-based environments, and lacked a more systematic investigation on understanding or abstracting common themes that underpin these. Having extended Triana to support our approach, we have opened the way to apply the described patterns and operators to Grid environments.

\section{Acknowledgements}

Simulation data courtesy of A. Nelson, N. White, P. Williams, and R. Philp, Galaxy Formation Group, De- partment of Physics and Astronomy, Cardiff University, UK. We would also like to thank Matthew Shields and Ian Wang for support on Triana programming. Special thanks are due to Ian Taylor for access to the Triana source code.

\section{References}

[1] Grid Computing Environments Working Group, See Web site at: http://www.computingportals.org/. Last visited: August 2002.

[2] A. Hoheisel, Fraunhofer Resource Grid - Grid Application Definition Language, Global Grid Forum, Edinburgh, July, 2002.

[3] Dan A. Marinescu, Internet Based Workflow Management: Towards a Semantic Web, Wiley, New York, 2002.

[4] Dan A. Marinescu, A Grid Workflow Management Architecture, Global Grid Forum Working Document (submitted), School of Electrical and Computer Engineering, University of Central Florida, Orlando, Florida 32816, USA.

[5] J. Mclaren, V. Sander and W. Ziegler, Grid Resource Allocation Agreement Protocol (GRAAP), See web site at: http://www.people.man.ac.uk/žzcgujm/GGF/graap-wg.html. See also the general Scheduling area of the Global Grid Forum, led by Bill Nitzberg and Jenny Shopf, at: http://wwwunix.mcs.anl.gov/ schopf/ggf-sched/. Last visited: August 2002.

[6] O.F. Rana and D. Jennings, Automating Performance Analysis from UML Design Patterns (Research Note), Proceedings of EuroPar 2000, Munich, Germany.

[7] UML Tools, See Web site at: http://www.cetus-links.org/oo uml.html\#oo_uml_utilities_tools. Last visited: August 2002.

[8] E. Gamma, R. Helm, R. Johnson and J. Vlissides, Design Patterns: Elements of Reusable Object-Oriented Software, Addison-Wesley, 1994.

[9] F. Buschmann, R. Meunier, H. Rohnert, P. Sommerlad and M. Stal, Pattern-Oriented Software Architecture: A System of Patterns, John Wiley \& Sons, 1998.

[10] I. Foster and C. Kesselman, The Globus Project: A Status Report, Proc. IPPS/SPDP '98 Heterogeneous Computing Workshop, 1998, pp. 4-18. Globus related publications can also be obtained from Web site at: http://www. globus.org/research/papers.html. Last visited: February 2003.

[11] S. Tuecke, K. Czajkowski, I. Foster, J. Frey, S. Graham and C. Kesselman, Grid Service Specification, Open Grid Service Infrastructure WG, Global Grid Forum, Toronto, Canada, February 2002. 
[12] G. von Laszewski, I. Foster, J. Gawor and P. Lane, A Java Commodity Grid Toolkit, Concurrency: Practice and Experience, 13, 2001.

[13] K. Czajkowski, I. Foster, N. Karonis, C. Kesselman, S. Martin, $\mathrm{W}$. Smith and S. Tuecke, A Resource Management Architecture for Metacomputing Systems, Proc. IPPS/SPDP '98 Workshop on Job Scheduling Strategies for Parallel Processing, 1998, pp. 62-82.

[14] Rajkumar Buyya, Grid Computing InfoCentre, See Web site at: http://www.gridcomputing.com/. Last visited: August 2002.

[15] G. Fox, D. Gannon and M. Thomas, A Summary of Grid Computing Environments, Concurrency and Computation: Practice and Experience, 2002.

[16] The Global Grid Forum. See Web site at: http://www. gridforum.org/. Last visited: August 2002.

[17] D.W. Walker, M. Li, O.F. Rana, M. Shields and Y. Huang, The Software Architecture of a Problem Solving Environment, Concurrency: Practice and Experience, December 2000.

[18] D. Abramson et al., A Tool for Distributed Parametric Modelling, See Web site at: http://www.csse.monash.edu.au/ davida/nimrod.html/. Last visited: August 2002.

[19] I. Taylor et al., TRIANA, See Web site at: http://www.triana. co.uk/. Last visited: October 2002.

[20] I. Taylor, O.F. Rana, R. Philp, I. Wang and M. Shields, Supporting Peer-2-Peer Interactions in the Consumer Grid, 8th International Workshop on High-Level Parallel Programming Models and Supportive Environments (HIPS) at IPDPS, Nice, France, April 2003. IEEE Computer Society Press.

[21] C. Lee, S. Matsuoka, D. Talia, A. Sussman, N. Karonis, G. Allen and J. Saltz, A Grid Programming Primer, Programming Models Working Group, Global Grid Forum meeting, Washington DC, July 16-18, 2001.

[22] I. Murray, Cole and Andrea Zavanella, Coordinating Hetero- geneous Parallel Systems with Skeletons and Activity Graphs, Journal of Systems Integration 10(2) (2001), 127-143.

[23] I. Murray, Cole, Algorithmic Skeletons: A Structured Approach to the Management of Parallel Computation, Pitman, 1989

[24] S. Gorlatch, Extracting and implementing list homomorphisms in parallel program development, Science of Computer Programming 33(1) (1998), 1-27.

[25] C.A. Herrmann and C. Lengauer, Transforming Rapid Prototypes to Efficient Parallel Programs, book chapter in Patterns and Skeletons for Parallel and Distributed Computing, A. Rabhi and Sergei Gorlatch, eds, Springer Verlag, 2002.

[26] The Common Component Architecture Forum. See Web site at: http://www.cca-forum.org/. Last visited: August 2002.

[27] E. Friedman-Hill, The Rule Engine for the Java Platform, See Web site at: http://herzberg.ca.sandia.gov/jess/. Last visited: August 2002.

[28] Michael Weber, Ekkart Kindler, The Petri Net Markup Language $(P N M L)$, See Web site at: http://www.informatik.huberlin.de/top/pnml/. Last visited: November 2002.

[29] Z. Nemeth and V. Sunderam, A Formal Framework for Defining Grid Systems, Proceedings of IEEE CCGrid 2002, Berlin, Germany

[30] D. Buchs and N. Guelfi, A Formal Specification Framework for Object-Oriented Distributed Systems, IEEE Transactions on Software Engineering 26(7) (July 2000).

[31] G. Di Marzo Serugendo, D. Mandrioli, D. Buchs and N. Guelfi, Adding Real-Time Constraints to Synchronised Petri Nets, Technical report 2000/341, EPFL, Lausanne, Switzerland, 2000.

[32] O.F. Rana and D.W. Walker, Service Design Patterns for Computational Grids, in: Patterns and Skeletons for Parallel and Distributed Computing, F. Rabhi and S. Gorlatch, eds, Springer, 2002. 

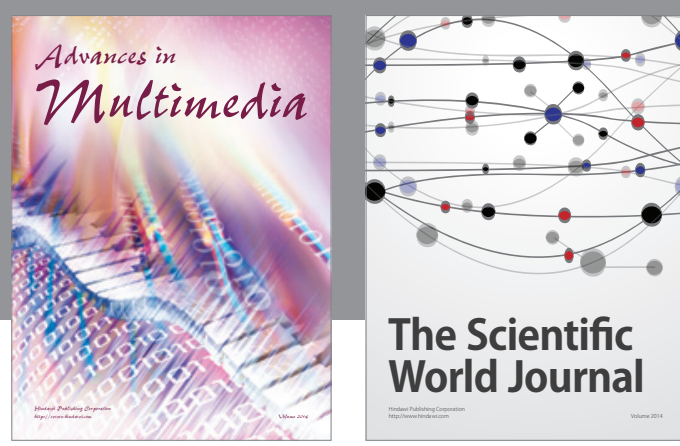

The Scientific World Journal
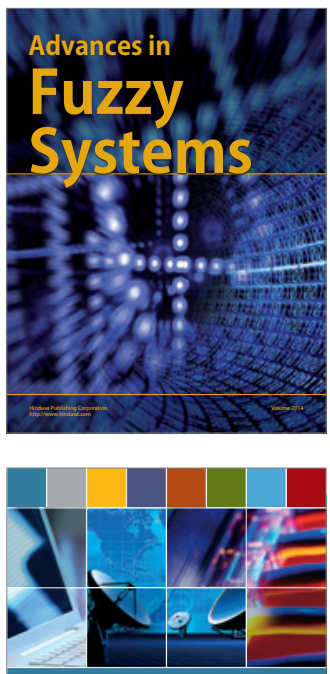

Computer Networks and Communications
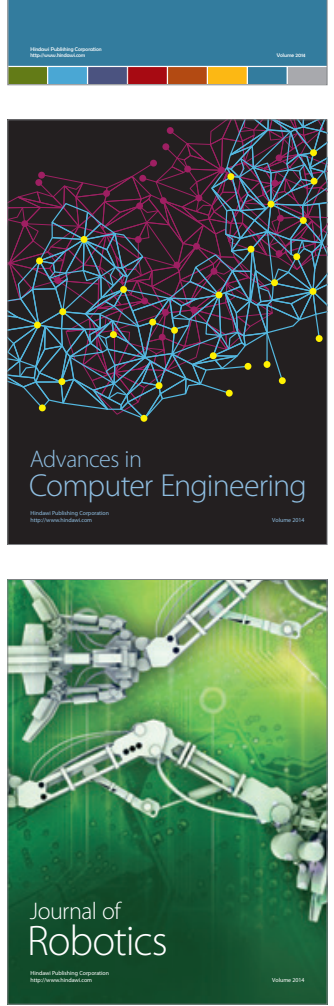
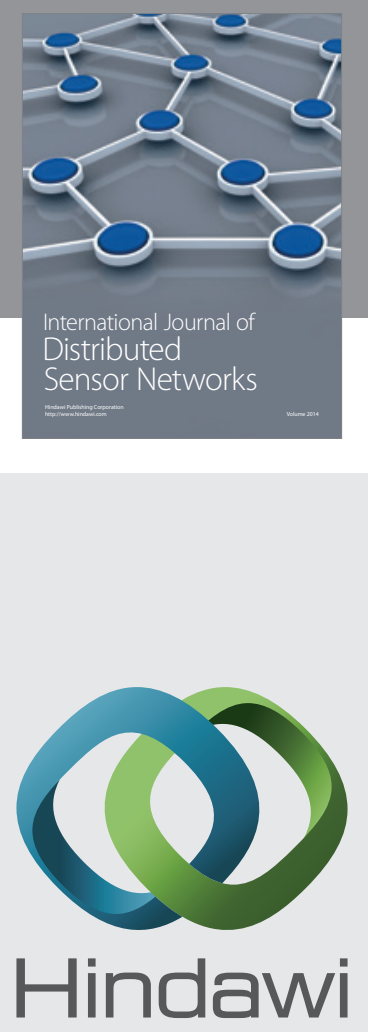

Submit your manuscripts at

http://www.hindawi.com
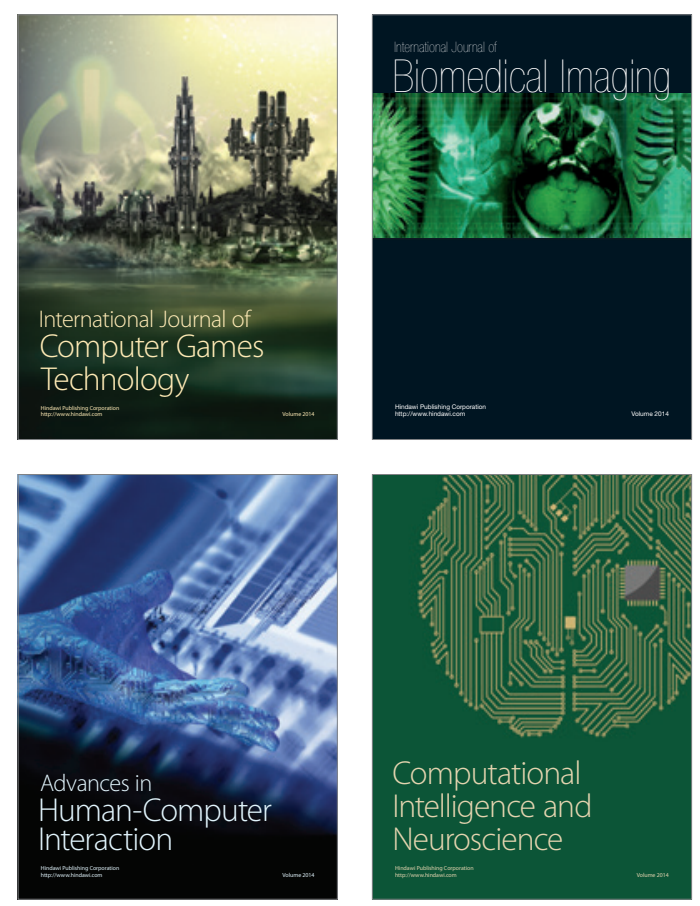
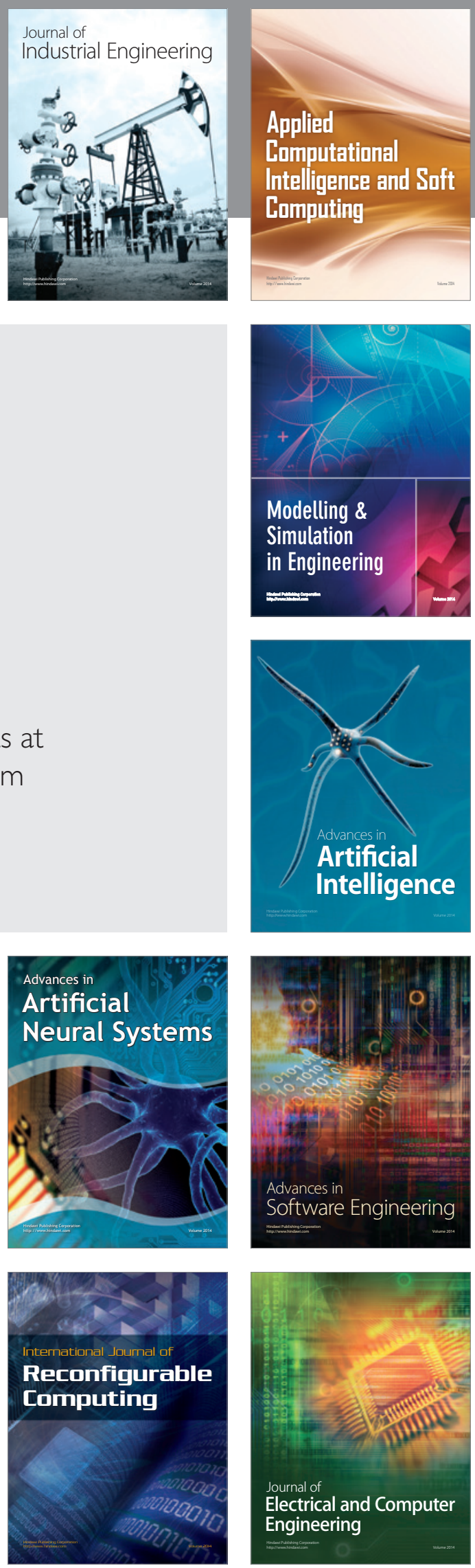